\title{
A systematic review on hand gesture recognition techniques, challenges and applications
}

\author{
Mais Yasen ${ }^{\text {Corresp., } 1}$, Shaidah Jusoh ${ }^{1}$ \\ ${ }^{1}$ Department of Computer Science, Princess Sumaya University for Technology, Amman, Jordan \\ Corresponding Author: Mais Yasen \\ Email address: mai20130045@std.psut.edu.jo
}

Background. With the development of today's technology, and as humans tend to naturally use hand gestures in their communication process to clarify their intentions, hand gesture recognition is considered to be an important part of Human Computer Interaction $(\mathrm{HCl})$, which gives the computers the ability of capturing and interpreting hand gestures, and executing commands afterwards. The aim of this study is to perform a systematic literature review for identifying the most prominent techniques, applications and challenges in hand gesture recognition.

Methodology. To conduct this systematic review, we have screened 560 papers retrieved from IEEE Explore published from the year 2016 to 2018, in the searching process keywords such as "hand gesture recognition" and "hand gesture techniques" have been used. However, to focus the scope of the study 465 papers have been excluded. Only the most relevant hand gesture recognition works to the research questions, and the well-organized papers have been studied.

Results. The results of this paper can be summarized as the following; the surface electromyography (sEMG) sensors with wearable hand gesture devices were the most acquisition tool used in the work studied, also Artificial Neural Network (ANN) was the most applied classifier, the most popular application was using hand gestures for sign language, the dominant environmental surrounding factor that affected the accuracy was the background color, and finally the problem of overfitting in the datasets was highly experienced.

Conclusions. The paper will discuss the gesture acquisition methods, the feature extraction process, the classification of hand gestures, the applications that were recently proposed, the challenges that face researchers in the hand gesture recognition process, and the future of hand gesture recognition. We shall also introduce the most recent research from the year 2016 to the year 2018 in the field of hand gesture recognition for the first time. 


\section{A Systematic Review on Hand Gesture Recognition}

3 Techniques, Challenges and Applications

4

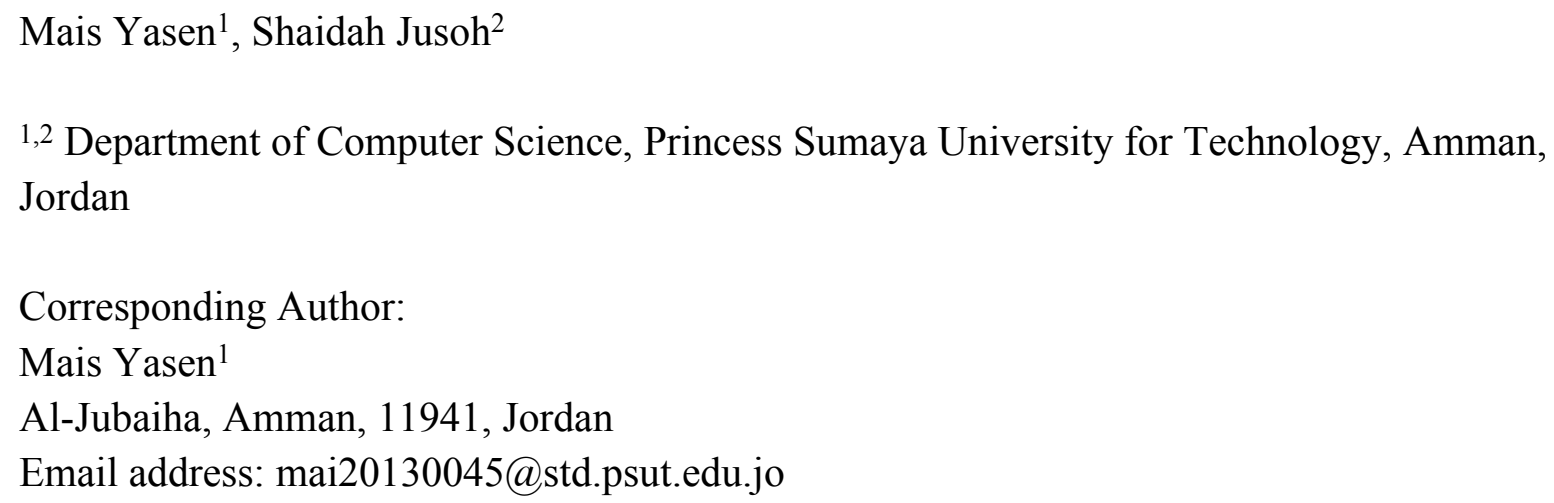

\section{Abstract}

Background. With the development of today's technology, and as humans tend to naturally use hand gestures in their communication process to clarify their intentions, hand gesture recognition is considered to be an important part of Human Computer Interaction (HCI), which gives the computers the ability of capturing and interpreting hand gestures, and executing commands afterwards. The aim of this study is to perform a systematic literature review for identifying the most prominent techniques, applications and challenges in hand gesture recognition.

Methodology. To conduct this systematic review, we have screened 560 papers retrieved from IEEE Explore published from the year 2016 to 2018, in the searching process keywords such as "hand gesture recognition" and "hand gesture techniques" have been used. However, to focus the scope of the study 465 papers have been excluded. Only the most relevant hand gesture recognition works to the research questions, and the well-organized papers have been studied. Results. The results of this paper can be summarized as the following; the surface electromyography (sEMG) sensors with wearable hand gesture devices were the most acquisition tool used in the work studied, also Artificial Neural Network (ANN) was the most applied classifier, the most popular application was using hand gestures for sign language, the dominant environmental surrounding factor that affected the accuracy was the background color, and finally the problem of overfitting in the datasets was highly experienced.

Conclusions. The paper will discuss the gesture acquisition methods, the feature extraction process, the classification of hand gestures, the applications that were recently proposed, the challenges that face researchers in the hand gesture recognition process, and the future of hand gesture recognition. We shall also introduce the most recent research from the year 2016 to the year 2018 in the field of hand gesture recognition for the first time. 


\section{Introduction}

41 A summary of the identification and selection of articles for inclusion in this review is presented

42

43

44

45

46

47

48

49

50

51

52

53

54

55

56

57

58

59

60

61

62

63

64

65

66

67

68

69

70

71

72

73

74

75

76

77

78

79 in Fig. 1, according to the PRISMA statement (Moher et al., 2009). Hand gestures are a nonverbal method of communication using the movements of the human hand. This method is used either on its own or with another parallel communication method such as speech (Adam, 2004). The movements of a hand on the air as shown in Fig. 2 (Rosalina et ,2017), is an example of a sign language using hand gestures. The representation of hand gestures is varying from reflecting a certain action to delivering a message that actually has a meaning (Adam, 2004). Hand

Gestures are considered to be culture dependent, which means one gesture can range from giving a complimentary meaning to a giving a highly offensive meaning (Adam, 1994).Hand gestures are widely distributed on different kinds of applications, ranging from applications that are connected to the safety of humans, such as using hand gestures in controlling and directing flights operations (landing and taking off), to applications that are made for pleasure purposes, such as using it in gaming (Mohini et al., 2015).

With the development of today's technology, and as humans tend to naturally use hand gestures in their communication process to clarify their intentions, hand gestures can play an important role in interchanging information between humans and computers (Aashni et al., 2017).

In Human-Computer Interaction (HCI) hand gestures have a wide number of applications that can guarantee the speed of communicating with the computer, give a user-friendly and aesthetic environment that would attract users, provide a nonphysical contact with the computer from a distance for user comfort and safety, and control complex and virtual environments in a much easier approach (Andrea, 2014).

On the other hand, hand gesture applications demand the user to be an expert and well trained at employing and understanding the meaning of different gestures (Samata \& Dr. K.S, 2015). Because many possible numbers of hand gestures, therefore, for each application, a different group of gestures is used to perform its operations. Also, from the computer perspective, the performance of recognizing hand gestures is affected by the environmental surroundings (such as light, background, distance, skin color) and the position and direction of the hand (Vaibhavi et al., 2014).

Perceptual computing is providing the computers with the ability of knowing what is happening around it (Bradley, 2015). In other words, the computer is capable of recognizing the different users and the different environmental factors occurring and existing in its surrounding. With that being said, hand gesture recognition is a type of perceptual computing user interface, that is used in HCI to give the computers the capability of capturing and interpreting hand gestures, and executing commands based on an understanding made on a certain gesture (Meenakshi, 2011). Hand gesture recognition technique steps vary from simple to complex applications. Generally, the steps are usually divided as the following: first hand gesture frame acquisition, then hand tracking, then feature extraction, and finally classification to reach the output gesture.

Hand gesture frame acquisition is to capture the human hand gesture by the computer (Ananya, 2015). Whereas hand tracking is the ability of the computer to trace the hand and separate it from

Peer] Comput. Sci. reviewing PDF | (CS-2018:12:33958:2:2:NEW 8 Aug 2019) 
80 the background and from the surrounding objects (Ananya, 2015). The features needed to be

81

82

83

84

85

86

87

88

89

90

91

92

93

94

95

96

97

98

99

100

101

102

103

104

105

106

107

108

109

110

111

112

113

114

115

116

117

118

119

extracted changes from one application to another, some of the features that could be taken into consideration are: fingers status, thumb status, skin color, alignments of fingers, and the palm position (Ananya, 2015). In artificial intelligence, machine learning enables the computers to learn without being explicitly programmed (Margaret, 2016). There are two types of learning; the process when algorithms reflect the gestures that has been learned in the past in training to new gestures is called supervised machine learning, and the process when algorithms draw inferences from the gestures is called unsupervised machine learning. Classification aims of building a model to classify new hand gestures based on previous training gestures. The research work in hand gesture recognition has been developing for more than 38 years (Prashan, 2014). In 1977, a system that detects the number of fingers bending using a hand glove was proposed by Zimmerman (Praveen \& Shreya, 2015). Furthermore, Gary Grimes in 1983 developed a system for determining whether the thumb is touching another part of the hand or fingers (Laura, Angelo \& Paolo, 2008). In 1990, despite the limited computer processing powers, the systems developed then gave promising results (Prashan, 2014).

The field of hand gesture recognition is very wide, and a big amount of work was conducted in the last 2 to 3 years. In this research, we survey the latest researches that were done on hand gesture recognition. We shall also compare the different techniques, applications, and challenges presented by the surveyed work. The reason why the most recent research articles from IEEE database were chosen to be studied is that we wanted to construct a valid base of the current situation and technologies of hand gesture recognition. Furthermore, the articles published by IEEE in the year of 2016 to 2018 were considered to increase the intensity and focus of this study, and because the recent works were not sufficiently studied before, where the older ones were studied and compared more such as in (Rafiqul \& Noor, 2012; Arpita. G. \& S., 2013; Deepali \& J., 2012). The contribution of this research can be summarized as the following:

1. Introducing the most recent researches from the year 2016 to the year 2018 in the field of hand gesture recognition for the first time.

2. Comparing the different techniques proposed, applications applied, and challenges discussed in the current available technology of hand gestures recognition.

The paper is structured as follows: Survey methodology section describes the research questions and methods used. The following section includes the results and analysis of the work studied. Then the next section discusses the future of hand gesture recognition. The last section concludes the research.

\section{Survey methodology}

This review is guided by the following 3 research questions:

1. What are the techniques used in hand gesture recognition?

2. What are the domains and applications that make use of hand gesture recognition?

3. What are the challenges in hand gesture recognition applications?

Peer) Comput. Sci. reviewing PDF | (CS-2018:12:33958:2:2:NEW 8 Aug 2019) 
120 The first step of this systematic literature review is gathering all retrieved documents from the

121

122

123

124

125

126

127

128

129

130

131

132

133

134

135

136

137

138

139

140

141

142

143

144

145

146

147

148

149

150

151

152

153

154

155

156

157

158

159

year 2016 to the year 2018, where the process of screening includes downloading the papers published on IEEE Explore, and reading their titles and abstracts. Two main keywords were used for the search process: hand gesture recognition and hand gesture techniques, other keywords were also used (such as hand gestures, hand gesture systems, hand gesture classification, hand gesture feature extraction, and sign language recognition) to include synonyms, where the keywords focused on the domain of research questions. The search process was performed by both authors (Mais Yasen and Shaidah Jusoh). A summary of the identification and selection of articles for inclusion in this review is presented in Fig. 1, according to the PRISMA statement (Moher et al., 2009). Literature included 1,045 studies. After removing duplicates 560 titles were screened. The duplication occurs because the same work was retrieved more than once when multiple keywords were used. Then 316 papers were excluded for having titles with no relevance to the review questions in general, where titles were scanned and processed by all authors to specify whether the works are pertinent to any chosen subcategory of hand gesture recognition. The next step was screening abstracts of all 244 retrieved documents. All 244 papers were reviewed and evaluated in reference to the research questions, where authors read the abstracts and evaluated whether they can find an answer to any of the core questions of this review, for example some of the papers excluded were discussing the electrical aspect of hand gesture acquisition methods, this process excluded 100 papers out of 244 . Full papers of 144 potentially relevant references were selected for further examination. After reading the full papers, 49 poorly organized documents that do not have a clear methodology justification were removed due to lack of validation and weakness of results justification, for example some of the works excluded did not explain why they chose their approach of acquisition or classification in comparison with all the possible approaches available in the recent technology, or did not explain why their proposed approach performed in the way they provide. Only 95 papers were selected to be included in this paper. Overall, the selection was made by the authors based on two criteria; relevance to the research questions, organization and form of writing of the papers studied. Classification of the selected paper is demonstrated in Table 1 which also shows the number of papers (intersected) included in each class and subclass. 180 papers were relevant to Hand Gesture Techniques, 53 papers were relevant to Hand Gesture Recognition Applications, and 31 papers were relevant to Hand Gesture Recognition Challenges.

\section{Results and Analysis}

The procedure of hand gesture recognition is conducted by executing several steps as illustrated in Fig. 3; image frame acquisition or gesture acquisition is to capture the human hand gesture image by the computer (Ananya, 2015). This could be done using vision-based recognition where no special gadgets are required, and a web camera or a depth camera is used, furthermore special tools can be utilized such as wired or wireless gloves that detect the movements of the user hand, and motion sensing input devices (Kinect from Microsoft, Leap Motion, ...etc.) that captures the hand gestures and motions.

Peer] Comput. Sci. reviewing PDF | (CS-2018:12:33958:2:2:NEW 8 Aug 2019) 
160 Hand tracking process is the ability of the computer to trace the user hand and separate it from

161

162

163

164

165

166

167

168

169

170

171

172

173

174

175

176

177

178

179

180

181

182

183

184

185

186

187

188

189

190

191

192

193

194

195

196

197

198

the background and from the surrounding objects (Ananya, 2015). This can be done using multiscale color feature hierarchies that gives the users hand and the background different shades of colors to be able to identify and remove the background, or by using clustering algorithms that are capable of treating each finger as a cluster and removing the empty spaces in-between them. The features extracted change from one application to another, some of the features that could be taken into consideration are: fingers status, thumb status, skin color, alignments of fingers, and the palm position (Ananya, 2015). These features and other features can be extracted using several methods available, such as Fourier descriptor method which captures the palm, the fingers and the finger tips, or centroid method which captures the essential structure of the hand. The features extracted are then sent to training and testing the classification algorithm (such as Artificial Neural Networks (ANN), K-nearest neighbor (KNN), Naive Bayes (NB), Support Vector Machine (SVM), ...etc.) to reach the output gesture, for example the output gesture in a simple case can contain two classes to detect only two gestures such as open and closed hand gestures. In this section we will be discussing the papers that were extracted in reference to the research questions in details, where Fig. 4 demonstrates the results of this review, answering each research question by showing the most popular method used in each subcategory.

\section{Hand Gesture Recognition Techniques}

The focus of our review in this section is on different gesture acquisition methods that has been used, the features considered for different applications and the feature extraction methods applied on the images to abstract those features, and finally the classification algorithms that has been recently used for classifying hand gestures.

\subsection{Gesture Acquisition Methods}

Image hand gesture acquisition, as illustrated in Fig. 5 from (Kinect, 2019) is to capture the human hand gesture image by the computer (Ananya, 2015). This could be done using vision-based recognition where no special gadgets are required and a web camera or a depth camera is used, furthermore special tools can be utilized such as wired or wireless gloves that detect the movements of the user hand, and motion sensing input devices (Kinect from Microsoft, Leap Motion, ...etc.) that capture the hand gestures and motions as showed in Fig. 5.

Several methods for hand gestures acquisition were discussed in the work studied using different acquisition tools, part of them used images that were captured in real time and others used previous images that were recorded in databases. Another classification to be considered is the nature of the acquisition which can be either a static hand gesture recognition where the gestures are represented by a constant image, or a dynamic hand gesture recognition were gestures are represented by the movement of the hand.

The first method is using vision-based hand gesture recognition to extract images which was proposed by (Weiguo, 2017; Ananyaa, 2017; Alvi, Fatema \&

Peer] Comput. Sci. reviewing PDF | (CS-2018:12:33958:2:2:NEW 8 Aug 2019) 
199

200

201

202

203

204

205

206

207

208

209

210

211

212

213

214

215

216

217

218

219

220

221

222

223

224

225

226

227

228

229

230

231

232

233

234

235

236

237

238

Mohammad, 2016; Shome, 2017). Where in (Weiguo et al., 2017) it was built in a real-time system.

In (Oinam et al., 2017) the authors implemented two different techniques of visionbased hand gesture recognition and one data glove-based technique. The visionbased techniques are static hand and real-time hand gesture recognition techniques. In data glove-based technique the glove had five flex sensors. Results showed that the vision-based technique was more stable and reliable compared to the data glovebased technique. Hand gestures are obtained by evaluating the contour captured from the image segmentation using a glove worn by the speaker in (Rosalina et al., 2017). Also, in (Danling, Yuanlong \& Huaping, 2016) they used a novel data glove called YoBu to collect data for gesture recognition.

The work in (P. D. S. H. \& Nimali, 2017) compared using a data glove to track the motion of the human hand using flex sensors, gyroscopes and vision data with Leap Motion Controller. Results showed that the Leap Motion Controller had a high repeatability and high potential for soft finger type applications. Furthermore, in (Eko, Surya \& Rafiidha, 2017; Shaun et al., 2017; Deepali \& Milind, 2016) the gestures are also captured using Leap Motion Controller.

In (S., K. \& M., 2017) They used a new Hand Gesture Control in Augmented Reality System (HGCARS) where the gesture recognition is performed using a secondary camera and the reality is captured using an IP camera, the virtual object is added to the video feed obtained from an IP camera and controlled by using the position and depth of hand, measured using a webcam. Moreover, the authors of (Tejashree, Salunke \& Bharkad, 2017; Rokhsana et al., 2017; Jessie et al., 2016; Anshal, 2017) used webcams for gathering input data.

Where in (Aditya et al., 2017) a hand gesture recognition on top-view hand images observed by a Time of Flight (ToF) camera in a car for touchless interactions inside a car was proposed. The help of image capturing devices installed on computer was implemented in (Nilima \& S., 2017). The authors of (Hafiz et al., 2017) presented a CMSWVHG (Control MS Windows via hand Gesture) to perform numerous windows actions using hand gestures using internal or external camera for taking input with the help of OpenCV. To control OS on the projected screen for a virtual mouse system without any hardware requirement one camera source was required (Rishabh et al., 2016). Also, data acquisition was done using Camera interfacing in (Ashish \& Aarti, 2016).

To detect static hand gesture RGB cameras and depth data were used by (Rytis \& Arunas, 2017; Chenyang, Xin \& Lianwen, 2017). Where in (Chenyang, Xin \& Lianwen, 2017) they tested their approach on Sheffield Kinect Gesture (SKIG). A static hand gesture recognition using Kinect depth sensor to capture color, and infrared and depth frames was applied by (Fuchang \& Hijian, 2016; Xiang, Lei \& Qiang, 2017; Jozef \& Slavomír, 2017; Sih-Huei et al., 2017; Yiwen et al., 2017).

Peer] Comput. Sci. reviewing PDF | (CS-2018:12:33958:2:2:NEW 8 Aug 2019) 
239

240

241

242

243

244

245

246

247

248

249

250

251

252

253

254

255

256

257

258

259

260

261

262

263

264

265

266

267

268

269

270

271

272

273

274

275

276

277

Moreover, the authors proposed a Kinect based real time dynamic hand gesture recognition technique in (Atharva \& Apurv, 2017; Devendrakumar \& S., 2016; Karthik et al., 2017).

A real time wearable hand gesture recognition system, which decoded the information from surface electromyography (sEMG) was proposed in (Jian et al., 2017; Jinxing, Jianhong \& Jun, 2017; David \& Donghan, 2018; Kuang-Yow et al., 2017; Jakub, Tomasz \& Piotr, 2017; Shunzhan et al., 2017; Andrés \& Marco, 2017). Also, a real time system based on the EMG with the surface electromyography measured by the commercial sensor Myo which is an armband placed on the forearm was proposed by (Marco et al., 2017a; Marco et al., 2017b; Karthik et al., 2017; Stefano et al., 2018). In (Yuhui, Shuo \& Peter, 2018; Yuta et al., 2017; Donq-Liang \& Wei-Shiuan, 2018; Ananta \& Piyanuch, 2016; Nabeel \& Rosa, 2017) a sensor-based wearable wristband was presented for static hand gestures. The authors in (Hari et al., 2016; Erhan, Hakan \& Baran, 2017) presented making use of the sensors in smartphones. The authors of (Yifan et al., 2018) used wearable devices such as VR/AR helmet and glasses in a gesture recognition system. A Doppler radar sensor with dual receiving channels was used to acquire a big database of hand gestures signals in (Jiajun \& Zhiguo, 2017). A gesture recognition system for human-computer interaction based on $24 \mathrm{GHz}$ radars in (Shengchang et al., 2017) and 2.4-GHz continuous radar in (Takuya et al., 2017) were used.

Moreover, an interface based on a mechanomyography (MMG) signal to capture arm movement and hand gesture was applied in (Yuntao et al., 2017; Piotr, Tomasz \& Jakub, 2017).

The work of (Ibrahim et al., 2018) proposed a hand gesture system using two monopole antennas to measure signatures at different locations, and the work of (Zengshan et al., 2018) developed a wireless sensing WiCatch, where the motion locus of the gesture is developed by constructing virtual antenna array based on signal samples.

\subsection{Feature Extraction}

Hand tracking process is the ability of the computer to trace the user hand and separate it from the background and from the surrounding objects (Ananya, 2015). This can be done using multi-scale color feature hierarchies that gives the users hand and the background different shades of colors to be able to identify and remove the background, or by using clustering algorithms that are capable of treating each finger as a cluster and removing the empty spaces in-between them. In this subsection we will be discussing the different features used by the different applications implemented in the reviewed work. Furthermore, the feature extraction methods used will be discussed too.

PeerJ Comput. Sci. reviewing PDF | (CS-2018:12:33958:2:2:NEW 8 Aug 2019) 
278

279

280

281

282

283

284

285

286

287

288

289

290

291

292

293

294

295

296

297

298

299

300

301

302

303

304

305

306

307

308

309

310

311

312

313

314

315

316

317

\subsubsection{Feature Extraction Methods}

Feature extraction methods are used in order to extract the useful information from the images that helps in the recognition process of gestures. These features can be extracted using several methods available, such as Fourier descriptor method which captures the palm, the fingers and the finger tips, or centroid method which captures the essential structure of the hand.

The work of (Haitham, Alaa \& Sabah, 2017) applied two methods of feature extraction, a contour hand and complex Alhzat. In (Weiguo et al., 2017) feature extraction module was applied using Discrete Fourier Transform (DFT) operations of histograms (vertical and horizontal). The proposed approach of (Tejashree, Salunke \& Bharkad, 2017) took the input data from a portable webcam then processed the images and after that extracted a histogram of gradients features. The objective of (Himadri et al., 2018) was to do segmentation of the hands using polygon approximation and approximate convex decomposition, then recording the unique features between various convex segments of the hand as a method of feature extraction.

Regional Contrast (RC) based salient object extraction algorithm, and a method using the color statistics of image were used in (Qingrui et al., 2017). To detect the start and end points of gestures a gesture spotting algorithm was applied in (Hari et al., 2016). Experiments of (Chenyang, Yingli \& Matt, 2016) followed 5 different feature extraction strategies; depth image sequence, body joints $\&$ facial landmarks, hand shapes \& facial expressions/attributes. In (C. Jose et al., 2017), digital image processing techniques were used to eliminate noise, to improve the contrast under different illumination, to separate the hand from the background and to cut the region containing the hand. Histogram of Oriented Gradients (HOG) was used in (Vimonhak, Aranya \& Somsak, 2017) for image processing to extract characteristics of the hand. In the work of (Nilima \& S., 2017), the Accurate End Point Identification method was implemented and applied on gesture images which were captured in varying backgrounds to detect edge points and branch points and it was also applied on blurred images containing multiple objects. The authors of (Isack, Zhongfu \& Jamal, 2017) employed higher order local autocorrelation (HLAC) feature extraction method.

The authors of (Xi, Chen \& Lihua, 2017) proposed using Wavelet Invariant Moments, the hand region was separated based on the depth information, and the wavelet feature was calculated by enforcing the wavelet invariant moments of the hand region, and the distance feature was extracted by calculating the distance from fingers to hand centroid.

Feature extraction like time, frequency and time-frequency were used in (Jiajun \& Zhiguo, 2017; Andrés \& Marco, 2017). Authors of (Chenyang, Xin \& Lianwen, 
318

319

320

321

322

323

324

325

326

327

328

329

330

331

332

333

334

335

336

337

338

339

340

341

342

343

344

345

346

347

348

349

350

351

352

353

354

355

356
2017) used a robust feature, path signature (PS) and its compressed version, log path signature (LPS) to extract effective features of hand gestures. Discrete wavelet transformation and singular value decomposition were used for features extraction, a genetic algorithm with effective fitness function was used to select optimal features by eliminating redundant and irrelevant features for improving the recognition performance of the system in (Rasel et al., 2017).

In (Rania, Mohammed \& Mahmoud, 2017) system for HGR that is based on dimensionality reducing the histogram of oriented gradients feature vectors by applying principal component analysis was implemented. A hand gesture recognition method based on salient feature point selection was proposed in (Yiwen et al., 2017), the shape feature of hand gesture was extracted from the contour, and the salient feature points were selected by a new algorithm to represent the hand gesture.

In experiments of (Vivek \& Ramesh, 2017) image processing techniques like outline investigating based on edge recognition, wavelet change, disintegration, widening, obscure disposal, and commotion end were used, they also used Histogram Orientation Gradient called HOG for shape highlight extraction and most vital part investigation for list of capabilities streamlining and diminishment. They also used a polygonal shape approximation strategy with a special chaincoding for shape-similarity matching in (Oyndrila et al., 2016). In (Vladislava, Predrag \& Goran, 2016) recognition was for 10 hand gestures, images were captured on two different backgrounds and with several space orientations, and histogram of Oriented Gradients method was applied for feature extraction. The Gaussian Mixture Models (GMM) based on human skin pixels and tracks segmented foreground using optical flow to detect hand swipe direction was proposed in (Srinidhi et al., 2016).

Authors of (Ananta \& Piyanuch, 2016) employed Daubechies wavelet transforms for feature extraction. In (Rajeshree \& Jayashree, 2016) they proposed a new method to find flow of hand for special signs using chain code, and recognition technique of signs (Dynamic digits) that is independent of size and color of hand using binary images. In (Ashish \& Aarti, 2016) feature extraction was done using Blob Detection and Contour extraction. In (Jessie et al., 2016) image processing techniques such as: color segmentation, visual-hand tracking, pre-processing, and feature extraction were used.

In (Ashish \& Aarti, 2016) features were extracted using Color jiltering and skin segmentation, convexity hull algorithm was implemented just for finger point detection and number recognition. Where in (Lalit \& Pritee, 2017), an acquisition module that spots the legitimate gesture trajectory by implementing pen-up and pen-down actions using depth thresholding and velocity tracking was proposed, 
357

358

359

360

361

362

363

364

365

366

367

368

369

370

371

372

373

374

375

376

377

378

379

380

381

382

383

384

385

386

387

388

389

390

391

392

393

394

395

396

the extracted trajectory is recognized using the equipolar signature (EPS) technique.

Conventional method was used in (Devendrakumar \& S., 2016) to utilize separation of hand from surrounding environment and then find palm points. Each hand part in (Shome, 2017) was modeled as a convex hull and pairwise distance between the parts was calculated using GJK-EPA algorithm. Principal Component Analysis (PCA) was used in (Marlon, Alistair \& Mohamed, 2016) to reduce the dimensionality and extract features of images of the human hand.

The work in (Anshal, Heidy \& Emmanuel, 2017) processed the hand gesture image by combining image segmentation and edge detection to extract morphological information, and frames were processed using the multi-modality technique used for processing individual characters. In (T. et al., 2017) to improve recognition rate of hand gestures, various image processing techniques were used such as Histogram Equalization, Median filtering, Average filtering, and Morphological filtering, feature extraction image matching was done using crosscorrelation co-efficient.

The proposed techniques in (Riya et al., 2017) relied on multiple representations namely HOG, GIST and BSIF, they used feature fusion which is the process of combining two feature vectors to obtain a single feature vector. Extraction of a series of features based on convex defect detection a model was proposed in (Soukaina et al., 2018), taking advantage of the close relationship of convex defect and fingertips.

\subsubsection{Features Extracted}

The features extracted change from one application to another, some of the features that could be taken into consideration are: fingers status, thumb status, skin color, alignments of fingers, and the palm position (Ananya, 2015).

There are several features that could be considered and extracted from the hand gestures which are highly dependent on the application. Skin color feature was used in (Weiguo et al., 2017; Xiang, Lei \& Qiang, 2017; Rokhsana et al., 2017). Hand gestures in (Himadri et al., 2018) were represented by hand shapes, orientations and movement of the hands, alignments of the fingers, and the palm position. In (David \& Donghan, 2018) open hand, wrist radial deviation, ulnar deviation, wrist extension, wrist flexion, and closed fist were considered. Scale, rotation, translation, illumination, noise and background were extracted in the work of (C. Jose et al., 2017). In (Yuta et al., 2017) finger movements were observed because the muscles and bones on the back of the hand are linked to the fingers, also skin deformation was measured by the distance between the device and skin with sensors.

The classifier in (Piotr, Tomasz \& Jakub, 2017) had 5 gestures (fist, pronation, supination, flexion, extension) and the feature vector consisted of 18 features: 5

Peer] Comput. Sci. reviewing PDF | (CS-2018:12:33958:2:2:NEW 8 Aug 2019) 
397

398

399

400

401

402

403

404

405

406

407

408

409

410

411

412

413

414

415

416

417

418

419

420

421

422

423

424

425

426

427

428

429

430

431

432

433

434

435

436

representing muscle activity (RMS) and 13 parameters corresponding to relative sensor orientation. A feature vector which was composed of wavelet invariant moments and distance feature was generated by the work of (Xi, Chen \& Lihua, 2017). The authors of (Shunzhan et al., 2017) extract five eigenvalues in the time domain. Geometric features such as area and centroid were extracted from each video frame to capture the trajectory of the moving hand and compare it with the training gestures in the experiments of (Atharva \& Apurv, 2017).

The features used in (P. D. S. H. \& Nimali, 2017) were: position, orientation, velocity and acceleration, bending angle of the fingers. To represent the global movement of hand skeleton the global motion features were extracted by (Xinghao et al., 2017). In experiments of (Sih-Huei et al., 2017) the hand region is recognized by using information about the skeleton of the segmented depth images, then a histogram of the oriented normal 4D (HON4D) and a histogram of oriented gradient (HOG) were extracted to represent the motion patterns.

For each hand gesture type, occlusion, light, shadow and background were considered as features in (Biyao et al., 2017). Where the work of (Anna, Sung \& Jae-Gon, 2018) presented a detection method that utilizes depth image obtained by incoming stereo image sequences and skin color information in a combined way, then a detected hand contours based on Bezier curve to provide an interoperable interface between a detection module and a recognition module was also presented, and a set of hand gestures with a combination of open fingers and rotational angles were used for the hand gesture recognition of their system. The proposed system (Oyndrila et al., 2016) identified hand-palm in video stream based on skin color and background subtraction scheme. Where in (Rishabh et al., 2016) glove tracking was done and then fingertips were detected with respect to centroid of the hand. Features used in (Ashish \& Aarti, 2016) were orientation, Centre of mass centroid, fingers status, thumb in positions of raised or folded fingers of hand. On the other hand, features extracted in (C. V \& Muzameel, 2017) were size, shape, color or texture.

Flexion, extension, abduction and grasp of forearm muscles features were used to detect four different movements of the wrist (Stefano et al., 2018). The features had to be able to characterize gestures, and invariant under translation and rotation of hand gesture to ensure reliable recognition in (Soukaina et al., 2018).

\subsection{Classification of Hand Gestures}

The features extracted are sent to training and testing the classification algorithm (such as Artificial Neural Networks (ANN), K-nearest neighbor (KNN), Naive Bayes (NB), Support Vector Machine (SVM), ...etc.) to reach the output gesture, for example the output gesture in a simple case can contain two classes to detect only two gestures such as open and closed hand gestures, or in sign language as shown in Fig. 2. 
437

438

439

440

441

442

443

444

445

446

447

448

449

450

451

452

453

454

455

456

457

458

459

460

461

462

463

464

465

466

467

468

469

470

471

472

473

474

475

476

The classification of the information extracted from the images of hand gestures is required to be able to recognize these gestures by the computer, there are several classification algorithms that can be used for this purpose.

The work of (Haitham, Alaa \& Sabah, 2017; Weiguo et al., 2017; Rosalina et al., 2017; Jakub, Tomasz \& Piotr, 2017; Piotr, Tomasz \& Jakub, 2017; Shunzhan et al., 2017; Hakan \& Baran, 2017; Vladislava, Predrag \& Goran, 2016-Deepali \& Milind, 2016; Ananta \& Piyanuch, 20 proposed using Artificial Neural Networks (ANN) for classification, where in (Jakub, Tomasz \& Piotr, 2017; Piotr, Tomasz \& Jakub, 2017) SoftMax output layer was used with feedforward ANN, in (Shunzhan et al., 2017; Hakan \& Baran, 2017; Alvi, Fatema \& Mohammad, 2016; Vladislava, Predrag \& Goran, 2016; Deepali \& Milind, 2016; Ananta \& Piyanuch, 2016) backpropagation training method was used, and the authors in (Jessie et al., 2016) used kohonen Self Organizing Maps as a type of ANN to classify data sets in unsupervised manner to convert hand gestures into Filipino words.

In (Chun-Jen et al., 2017) a synthetically-trained neural network was used for 3D hand gesture identification. The training process of a deep-learning neural network required a large amount of training data. The authors of (Chenyang, Xin \& Lianwen, 2017) proposed using the LPSNet, an end-to-end deep neural network for hand gesture recognition with novel log path signature features. In (Yifan et al., 2018; Biyao et al., 2017) they proposed using deep neural networks for classification. The authors in (Sungho \& Wonyong, 2016) came up with two dynamic hand gesture recognition techniques using low complexity recurrent neural network (RNN) algorithms for wearable devices, the first was based on video signal and uses convolutional neural network (CNN) with RNN for classification, and the other used accelerometer data and applied RNN for classification. Whereas in (Xinghao et al., 2017) a bidirectional recurrent neural network (RNN) with the skeleton sequence to augment the motion features for RNN were used.

Deep convolutional neural network was used for classification in (Ibrahim et al., 2018; David \& Donghan, 2018; Javier, Paula \& Robinson, 2017; C. et al., 2017; Aditya et al., 2017; Yuntao et al., 2017; Peijun et al., 2017; Jozef \& Slavomír, 2017; Jiajun \& Zhiguo, 2017; Takuya et al., 2017; Fabian, 2017), where in (Aditya et al., 2017) the authors aimed to improve the detection of hand-gestures by correcting the probability estimate of a Long-Short-Term Memory (LSTM) network by pose prediction performed by a Convolutional Neural Network (CNN). They used Principal Component Analysis (PCA) as a training procedure to reduce the labelled data of hand pose classification to perfect the initialization of weights for the CNN. The authors in (Fuchang \& Hijian, 2016; Tejashree \& S., 2017; Marlon, Alistair \& Mohamed, 2016) used K-nearest neighbor (KNN) classifier to recognize hand gestures. The work presented in (Kuang-Yow et al., 2017) classified the recognition using k-Nearest Neighbor (KNN) and Decision Tree algorithms combination.

Peer] Comput. Sci. reviewing PDF | (CS-2018:12:33958:2:2:NEW 8 Aug 2019) 
477

478

479

480

481

482

483

484

485

486

487

488

489

490

491

492

493

494

495

496

497

498

499

500

501

502

503

504

505

506

507

508

509

510

511

512

513

514

515
Support vector machine (SVM) was used for classification in (Yuhui, Shuo \& Peter, 2018; Chenyang, Yingli \& Matt, 2016; Yuta et al., 2017; Xi, Chen \& Lihua, 2017; Rasel et al., 2017; S. \& M., 2017; Zengshan et al., 2018; Ashish \& Aarti, 2016; Zhiwen et al., 2017; Stefano et al., 2018). Decision tree was used to classify the gestures in (Shengchang et al., 2017). To recognize the patterns in (Jian et al., 2017), they used a modified deep forest algorithm. In (Shaun et al., 2017; Riya et al., 2017) they used a random regression forest algorithm for classification.

In (Jinxing, Jianhong, \& Jun, 2017) the hand gesture was modeled and decomposed by the use of Gaussian Mixture Model-Hidden Markov Models (GMMHMM), GMMs are used as sub-states of HMMs to decode sEMG feature of gesture.

Moreover, a hand gestures recognition system was implemented using the incorporation of Bhattacharyya divergence into Bayesian sensing hidden Markov models (BS-HMM) in (Sih-Huei et al., 2017). Also, in (Devendrakumar \& S., 2016) Hidden Markov Model was used for classification.

The work of (Hari et al., 2016; Atharva \& Apurv, 2017; Yiwen et al., 2017; Washef, Kunal \& Soma, 2016) presented using the dynamic time warping algorithm for classification. Whereas (Marco et al., 2017a; Marco et al., 2017b) used the k-nearest neighbor rule together with the dynamic time warping algorithm for classification. Naive Bayes was applied as the training method for classification of (Eko, Surya \& Rafiidha, 2017). Multiple linear discriminant analysis (LDA) classifier was adopted to classify different hand gestures in (Isack, Zhongfu \& Jamal, 2017; Yuefeng et al., 2016).

Classification of digits gesture from 11 to 20 was done using Principal component analysis by (Rajeshree \& Jayashree, 2016). The work (Danling, Yuanlong \& Huaping, 2016) used extreme learning machine (ELM) for gesture recognition. The work presented in (Himadri et al., 2018) used Support Vector Machine (SVM), Artificial Neural Network, Naive Bayes and K-Nearest Neighbor (K-NN) classifiers as the training methods to recognize the features extracted. Whereas in (Ananyaa et al., 2017) the best classification accuracy was achieved using Euclidean distance and Eigen vector, but this result is for a very small dataset, the best result was a dataset containing nearly 720 images that used Support vector machine for classification of images, also using Artificial Neural Network got an accuracy of 89.48\%.

Furthermore, Multi-class Support Vector Machine (SVM) and k-Nearest Neighbors (KNN) classifiers were used to classify the hand gestures in (Rania, Mohammed \& Mahmoud, 2017), experiments showed that the accuracy of KNN classifier were better than SVM classifier. In (Nabeel \& Rosa, 2017) Support Vector Machine (SVM), Decision Tree (DT), K-Nearest Neighbors (KNN), and Linear Discriminant Analysis (LDA) were compared in classification performance, and results showed that LDA got the highest accuracy. 
516

517

518

519

520

521

522

523

524

525

526

527

528

529

530

531

532

533

534

535

536

537

538

539

540

541

542

543

544

545

546

547

548

549

550

551

552

553

\section{Hand Gesture Recognition Applications}

Hand gestures can be used as a natural communication with the computer, with today's technologies the number of applications that could apply hand gesture recognition is rapidly increasing. In this section we will be discussing the most recent applications that were presented in the years 2016 to 2018 .

\subsection{Sign Language}

Sign languages, as illustrated in Fig. 2, use manual communication to explain a certain meaning. This can include using hand gestures, movements, fingers orientation to deliver a certain word or meaning. The work of (Weiguo et al., 2017; Chenyang, Yingli \& Matt, 2016; Rasel et al., 2017; Shaun et al., 2017; Deepali \& Milind, 2016; Nabeel \& Rosa, 2017; Anshal, Heidy \& Emmanuel, 2017) proposed using hand gesture recognition for American Sign Language (ASL) for people who are hearing impaired . Where in (Shaun et al., 2017) they did not evaluate the letters that are dynamic (like: $\mathrm{j}$ and $\mathrm{z}$ ). In (Nabeel \& Rosa, 2017) 36 gestures were studied including 26 ASL alphabets and 10 ASL numbers. In (Anshal, Heidy \& Emmanuel, 2017) two different translation paradigms; English characters (alphabet) and complete words or phrases were proposed. An alternative to talking for deaf $\&$ dumb people who have hearing or speech problems using sign language recognition and hand gestures was proposed in (Himadri et al., 2018; Oinam et al., 2017; S. \& M., 2017; Vivek \& Ramesh, 2017; Oyndrila et al., 2016; Ashish \& Aarti, 2016; Rishabh et al., 2016). Whereas in (Rosalina et al., 2017) a sign language that consists of the alphabetic from " $A$ " to "Z", the numbers from " 0 " to " 9 ", and some additional punctuation marks like "Period", "Question Mark", and "Space" using static hand gesture recognition was produced.

The work of (C. Jose et al., 2017) used the alphabet of sign language of Peru (LSP). Also, (Vimonhak, Aranya \& Somsak, 2017) proposed a technique for the recognition of Lao alphabet sign language for the deaf people. In (Eko, Surya \& Rafiidha, 2017) Indonesian Sign Language System (SIBI) was applied for normal people to learn sign language and communicate to people with hearing disability. Moreover in (Donq-Liang \& Wei-Shiuan, 2018) the authors used 29 Turkish fingerspelling signs. A framework for recognizing Sinhala sign language gestures and translating them in to natural language for hearing \& speaking impaired people was proposed in (Vladislava, Predrag \& Goran, 2016). The authors of (Jessie et al., 2016) converted hand gestures into Filipino words. The work presented in (Marlon, Alistair \& Mohamed, 2016) used the alphabet of Irish Sign Language. Finally, Indian Sign Language (ISL) hand gesture recognition system was introduced in (Riya et al., 2017). 
554

555

556

557

558

559

560

561

562

563

564

565

566

567

568

569

570

571

572

573

574

575

576

577

578

579

580

581

582

583

584

585

586

587

588

589

590

591

592

593

\subsection{Robotics}

Hand gestures can also be employed in the process of building robotics, as illustrated in Fig. 6 (elecbits, 2019) which shows the use of a glove to control a robotic vehicle. A recognition frame of continuous hand gestures of upper-limb exoskeleton robot was proposed in (Jinxing, Jianhong, \& Jun, 2017). In (Yuhui, Shuo \& Peter, 2018) they had three groups of hand gestures: six wrist gestures, five single finger flexions, and ten Chinese number gestures that were built to control a robotic arm. The authors of (Sungho \& Wonyong, 2016) came up with two dynamic hand gesture recognition techniques using low complexity recurrent neural network (RNN) algorithms for a wearable device.

The experiments of (Xiang, Lei \& Qiang, 2017) produced a control system for robotic wheelchair for the aged and the disabled, which contained two parts: gesture interaction and intelligent wheelchair. Also, the approach of (Javier, Paula \& Robinson, 2017) allowed the consequent execution of a robotic agent for the delivery of objects. In (Yuntao et al., 2017) the authors introduced a wearable sensor suite fusing arm motion and hand gesture recognition for operator control of UAVs. The authors of (Yuta et al., 2017) proposed a wearable device with photo-reflective sensors arranged in an array to recognize hand gestures by measuring the skin deformation of the back of the hand. Also, in (Danling, Yuanlong \& Huaping, 2016) hand gestures recognition was used in human-robot interaction (HRI). In experiments of (Devendrakumar \& S., 2016), continuous trajectory path made by hand over a period of time was considered for real time dynamic gesture recognition purpose using Kinect sensor for controlling robotic arm. A hand gesture-based control design was proposed for mobile robots in (Hang et al., 2017), where mobile robots can move according to the signals encoded by hand gestures. Work of ( $\mathrm{T}$. et al., 2017) dealt with a robotic arm using hand gestures for many applications such as automation, medical applications and gaming.

\subsection{Others}

A static hand gesture recognition in a real-time human-computer interaction application was proposed in (Tejashree, Salunke \& Bharkad, 2017) to control the Power Point presentations from a distance. A human-computer interaction based on hand gesture in a projector-camera system was presented in (Qingrui et al., 2017). Also, real-time hand gesture recognition for flight control of multiple quadrotors was applied in (David \& Donghan, 2018). The authors of (Javier, Paula \& Robinson, 2017) presented the design of a convolutional neural network architecture using the MatConvNet library in order to achieve the recognition of 2 classes: "open" and "closed" and "unknown". The approach studied in (Marco et al., 2017) could be used in multiple applications in medical and engineering fields.

The study of (Aditya et al., 2017) showed hand gesture recognition on top-view hand images observed by a Time of Flight (ToF) camera in a car for touchless interactions 
594

595

596

597

598

599

600

601

602

603

604

605

606

607

608

609

610

611

612

613

614

615

616

617

618

619

620

621

622

623

624

625

626

627

628

629

630

631

632

633

inside a car. In (Peijun et al., 2017) they used seven kinds of hand gestures that can command a consumer electronics device, such as mobile phones and TVs. The authors in (S., K. \& M., 2017) proposed an Augmented Reality (AR) to merge the virtual world into the real world and to enhance the perception of the real world by adding 2-D virtual objects.

The experiments of (Yu et al., 2017) described a method of hand gesture recognition using Principle Component Analysis (PCA) implemented in Android phone. A realtime hand gesture recognition system was proposed using electromyography (EMG) in the field of medicine and engineering, with a higher number of gestures to recognize in (Marco et al., 2017). The work of (Rokhsana et al., 2017) presented a hand gesture-based computer mouse control system. The authors of (Hafiz et al., 2017) presented a CMSWVHG (Control MS Windows via hand Gesture) to perform numerous windows actions using hand gestures.

The work discussed in (Anna, Sung \& Jae-Gon, 2018) presented a method to detect and to recognize hand gestures for generating hand gesture-based commands to control the media consumption in smart glasses. Hand gesture recognition or finger angle prediction for Ultrasound imaging was proposed in (Yuefeng et al., 2016). A hand gesture recognition technique on a smartphone using Google Cardboard (GC) and Wearality2 in phones was applied in (Srinidhi et al., 2016).

A real-time hand gesture recognition technique for presentation was proposed in (Rishabh et al., 2016), to control OS on the projected screen for a virtual mouse system without any hardware requirement. Hand-gesture-based commands were proposed in (Lalit \& Pritee, 2017) to replace touch and electromechanical input panels using vision-based mid-air character input.

Gesture recognition system was proposed in (C. V \& Muzameel, 2017), Mudra is an expressive form of gesture that is mainly used in Indian classical dance form where the gesture is in visual form to connect with the audience. The authors in (Zhiwen et al., 2017) presented a real-time hand gesture recognition by using Kinect sensor, to control mouse by user hands for operations such as 'clicking', 'dragging' and 'dropping', and engaged/disengaged gestures. Gesture recognition in (Karthik et al., 2017) was used for Bio Robotics, the paper focused on presenting a sensor based human gesture recognition for the Hand Cricket game.

\section{Hand Gesture Recognition Challenges}

The process of hand gestures recognition consists of a set of complex steps that has many possible difficulties that could stand in the way of having accurate recognition. In this section we will discuss the major environmental surroundings challenges and the training and testing dataset challenges that could occur.

\subsection{Environmental Surroundings}

In (Fuchang \& Hijian, 2016) the environmental background, light and rotation, translation and scale change proposed a difficulty; the authors used depth data to

Peer] Comput. Sci. reviewing PDF | (CS-2018:12:33958:2:2:NEW 8 Aug 2019) 
634

635

636

637

638

639

640

641

642

643

644

645

646

647

648

649

650

651

652

653

654

655

656

657

658

659

660

661

662

663

664

665

666

667

668

669

670

671

672

673 help in hand separation and to accomplish a synchronized color and depth images in Kinect. Different light brightness (dull, medium, and bright) conditions were tested in (Tejashree, Salunke \& Bharkad, 2017) to overcome their problem, the results of the bright light level gave the best accuracy as expected. Furthermore, the results of (Oinam et al., 2017) showed that the vision-based techniques gave an accuracy of $100 \%$ in bright lighting condition with a white background.

The work of (Qingrui et al., 2017) showed that it was difficult to achieve proper accuracy when having complex backgrounds, variable external illumination, and shadows of hand gesture. The authors of (Hari et al., 2016) presented a continuous hand gestures recognition technique using three-axis accelerometer and gyroscope sensors in smartphones, a gesture coding algorithm was also used to reduce the influence of unstableness of the user hand.

Digital image processing techniques were used in (C. Jose et al., 2017) to eliminate noise, to improve the contrast under different illumination, to separate the hand from the background and to cut the region containing the hand, where results of images with complex background got low accuracy.

In (Rytis \& Arunas, 2017) the authors suggested that RGB cameras can be used to detect hand, but it has limited applications because hand detection was hard in some lighting conditions or in different skin colors, using depth camera data was better in distinguishing hands in front of camera.

Study of (Peijun et al., 2017) suggested that spatial localization of the hands when it contains background, human face, and human body could be a challenging task, where the results achieved an accuracy of $97.1 \%$ in the dataset with simple backgrounds and $85.3 \%$ in the dataset with complex backgrounds.

The authors of (Yu et al., 2017) were capable of solving the problems that faced them such as different size of gesture image captured, different angle of gesture's rotation and flipped gesture. After experiments of (Atharva \& Apurv, 2017) they found that if there was a larger object than the hand that the Kinect captures, wrong results will be achieved. The results of (Hafiz et al., 2017) were highly affected by the background noise where they achieved an accuracy of $82.52 \%$.

In experiments of (Yifan et al., 2018) and to strengthen the recognition accuracy, and as the dataset used contained more than 24,000 gesture and 3,000,000 frames for both color and depth modalities from 50 subjects, they tested 83 different static and dynamic gestures with 6 diverse indoor and outdoor scenes respectively with variation in background and illumination, they also tested when people perform gestures while they are walking and they achieved an acceptable accuracy.

In (S. \& M., 2017) The authors used complex backgrounds with respect to the skin color, and results showed a disadvantage in this system which was the lighting conditions. The results of (Rania, Mohammed \& Mahmoud, 2017) showed that the proposed algorithm achieved recognition rate of $97.69 \%$ under different hand poses 
674

675

676

677

678

679

680

681

682

683

684

685

686

687

688

689

690

691

692

693

694

695

696

697

698

699

700

701

702

703

704

705

706

707

708

709

710

711

712

and complex background with changes in lightning, moreover their system was robust to rotation, scale, translation, and lighting.

Also, the results of (Yiwen et al., 2017) showed that their method was adjusting to translation, rotation scaling and articulated deformation. Time of Flight (ToF) data was used in (Fabian et al., 2017) which tends to be overly noisy depending on various factors such as illumination, reflection coefficient and distance that were studied in their research process. The system of (Washef, Kunal \& Soma, 2016) overcame the limitations of a glove-based approach and the vision-based approach concerning different illumination conditions, background complexity and distance from camera. The system in (Lalit \& Pritee, 2017) was applicable for rotation, scale, and translation variations, directions, and the dataset contained digits, alphabets, and symbols.

\subsection{Training and Testing Data}

The training and testing data problems can be classified as either having a small set of gestures that are insufficient for recognition training or having a large set of gestures that are imbalanced leading to a low recognition accuracy. The training data of (Haitham, Alaa \& Sabah, 2017) had only 25 images and the results reached an accuracy of $82.2 \%$. In (Weiguo et al., 2017) they trained their system using 24 static American Sign Language (ASL), and their accuracy reached 93.3\%. Whereas in (Fuchang \& Hijian, 2016) to avoid the problem of having imbalanced data and images they used detailed features.

To overcome the problem of having more than one meaning and move to represent a gesture the authors of (Oinam et al., 2017) used vision-based hand gesture recognition systems and data glove-based hand gesture recognition systems. In (Jian et al., 2017) 16 hand gestures include 10 dynamic and 6 static gestures were used, and results showed that gestures were correctly recognized and the accuracy was $96 \%$.

The work in (Ibrahim et al., 2018) tested the system using ten hand gestures achieving their goal which is to maximize the recognition accuracy using Pre-trained networks. The data of (Javier, Paula \& Robinson, 2017) contained six architectures with variance in the hyperparameters and depth, their results achieved good accuracy. Classification of 24 hand gestures to improve the rate of recognition accuracy was proposed in (C. Jose et al., 2017) reaching $96.20 \%$ for simple backgrounds.

In (Aditya et al., 2017) five hand poses with nine class hand gestures were used reaching an accuracy of $89.50 \%$ as a result. The work of (Vimonhak, Aranya \& Somsak, 2017) used 54 Lao alphabets in the experiments, testing data had 540 gestures from 4 individuals, it was difficult to maintain constant speed of hand gestures, thus the recognition rate was $79 \%$.

PeerJ Comput. Sci. reviewing PDF | (CS-2018:12:33958:2:2:NEW 8 Aug 2019) 
713

714

715

716

717

718

719

720

721

722

723

724

725

726

727

728

729

730

731

732

733

734

735

736

737

738

739

740

741

742

743

744

745

746

747

748

749

750

751

The main difficulties of the hand gesture recognition (Andrés \& Marco, 2017) with EMG using machine learning are: the noisy behavior of EMG signal, and the small number of gestures per person relative to the number of generated data by each gesture (overfitting). Whereas, in (Chun-Jen et al., 2017) the training process of a deep-learning neural network required a large amount of training data, they combined a large set of computer-generated 3D hand images with few real camera images to form the training data set, the testing and training sets had 24 classes of hand gestures, and the results accuracy was $77.08 \%$.

The authors of (Deepali \& Milind, 2016) considered 26 different alphabets of American Sign Language, with a total of 520 samples (consisting of 20 samples of each alphabet), and results gave an accuracy of $96.15 \%$. In study of (Stefano et al., 2018) to avoid correlation between training and testing datasets the Leave One Subject Out (LOSO) cross-validation technique was used, and the accuracy was $92.87 \%$.

\section{The Future of Hand Gesture Recognition}

Despite the high performance of some of the recent methods discussed in this research, the hand gesture recognition is still an evolving topic that needs more experiments. Hand gesture recognition method also needs to be extended to cover all of the areas of information technology and artificial intelligence, such as tablets, smartphones, gaming consoles, smart televisions, laptops and desktops (Hexa, 2017).

It is no doubt that hand gesture recognition is capable of enabling natural communication and intelligence into applications that humans use every day. Hand gesture recognition is employing the principle of perceptual computing and changing the methods of human computer interaction (HCI) making them less complex and more enjoyable.

Applications such as home control systems, healthcare systems, gaming technologies, automobiles, televisions, home automations, and robotics are expected to be able to use hand gesture recognition to represent the communication between the user and the devices (Hexa, 2017). Furthermore, some of the applications are very sensitive and in need of having a high recognition accuracy almost close to $100 \%$, to be able to use them without causing any damage or danger to human lives; such as applications of the health field, the transportation field, and the flight operation field.

Hand gesture recognition was recently applied in some gaming consoles has increased the sales rate of these consoles (such as Xbox and PlayStation), it is expected to keep growing more and more over time. Smartphones are expected to experience the highest growth of hand gesture recognition (Hexa, 2017). Smart televisions are also expected to experience a growth in this topic and increase the purchasing rate of the latest technology using hand gestures (Hexa, 2017). The topic is expected to grow over 28\% from the year 2017 to 2024 (Hexa, 2017). 


\section{Conclusions}

753 This paper had successfully presented the most prominent techniques, applications, and

754 challenges in hand gesture recognition. These include the gesture acquisition methods, the

755 feature extraction process, the classification of hand gestures, the applications that were recently

756 proposed in the field of sign language, robotics and other fields, the environmental surroundings

757 challenges and the datasets challenges that face researchers in the hand gesture recognition

758 process, and the future of hand gesture recognition.

759 The results of this paper can be summarized as the following; the surface electromyography

760 (sEMG) sensors with wearable hand gesture devices were the most acquisition tools used in the

761 works studied, also the Artificial Neural Network (ANN) was the most applied classifier, the

762 most popular application was using hand gestures for sign language, the dominant environmental

763 surrounding factor that affected the accuracy was the background color, and the common

764 problem found in many of the studies was overfitting in the datasets.

765

766

\section{References}

768 Aashni Hariaa. Archanasri Subramaniana. Nivedhitha Asokkumara. Shristi Poddara. Jyothi

769 SNayak. 2017. International Conference on Advances in Computing \& Communications.

770 Science Direct. 115: 367-374.

771 Adam Kendon. 1994. Human gestures Tools. Language and Cognition in Human Evolution.

772 Cambridge: Cambridge University Press.

773 Adam Kendon. 2004. Gesture: Visible Action as Utterance. Cambridge: Cambridge University

774 Press.

775 Aditya Tewari. Bertram Taetz. Frederic Grandidier. Didier Stricker. 2017. A Probabilistic

776 Combination of CNN and RNN Estimates for Hand Gesture Based Interaction in Car.

777 International Symposium on Mixed and Augmented Reality ISMAR-Adjunct: 1-6.

778 Alvi Mahadi. Fatema Tuj Johora. Mohammad Abu Yousuf. 2016. An Efficient Approach of

779 Training Artificial Neural Network to Recognize Bengali Hand Sign. International Conference

780 on Advanced Computing IACC: 152-157.

781 Ananta Srisuphab. Piyanuch Silapachote. 2016. Artificial Neural Networks for Gesture

782 Classification with Inertial Motion Sensing Armbands. 10 Conference TENCON: 1-5.

783 Ananya Choudhury. Anjan Kumar Talukdar. Kandarpa Kumar Sarma. 2015. A Review on

784 Vision-Based Hand Gesture Recognition and Applications. IGI Global: 261-286.

785 Ananyaa Sharrma. Ayush Khandelwal. Kavleen Kaur. Shivani Joshi. Richa Upadhyay. Sameer

786 Prabhu. 2017. Vision based static hand gesture recognition techniques. International Conference

787 on Communication and Signal Processing ICCSP: 0705-0709. 
788 Andrea Attwenger. 2014. Advantages and Drawbacks of Gesture-based Interaction. Computer 789 Science - Miscellaneous: 1-11.

790 Andrés G. Jaramillo. Marco E. Benalcázar. 2017. Real-time hand gesture recognition with EMG 791 using machine learning. Ecuador Technical Chapters Meeting ETCM: 1-5.

792 Anna Yang. Sung Moon Chun. Jae-Gon Kim. 2018. Detection and Recognition of Hand Gesture 793 for Wearable Applications in IoMT. International Conference on Advanced Communication 794 Technology ICACT: 1-1.

795 Anshal Joshi. Heidy Sierra. Emmanuel Arzuaga. 2017. American Sign Language Translation 796 Using Edge Detection and Cross Correlation. Colombian Conference on Communications and 797 Computing COLCOM: 1-6.

798 Arpita Ray Sarkar. G. Sanyal. S. Majumder. 2013. Hand Gesture Recognition Systems: A 799 Survey. International Journal of Computer Applications: 71: 26-37.

800 Ashish S. Nikam. Aarti G. Ambekar. 2016. Bilingual Sign Recognition Using Image Based Hand 801 Gesture Technique for Hearing and Speech Impaired People. International Conference on 802 Computing Communication Control and automation ICCUBEA: 1-6.

803 Ashish S. Nikam. Aarti G. Ambekar. 2016. Sign Language Recognition Using Image Based 804 Hand Gesture Recognition Techniques. International Conference on Green Engineering and 805 Technologies IC-GET: 1-5.

806 Atharva Ajit Kadethankar. Apurv Dilip Joshi. 2017. Dynamic hand gesture recognition using 807 Kinect. Innovations in Power and Advanced Computing Technologies i-PACT: 1-3.

808 Biyao Shao. Yifeng Xie. Hongnan Yang. Yatong Jiang. Chenggang Yan. Hongtao Xie. Yangang 809 Wang. 2017. A New Dataset for Hand Gesture Estimation. Global Conference on Signal and 810 Information Processing GlobalSIP: 1388-1392.

811 Bradley L. Jones. 2015. It's Happening Now: Perceptual Computing is Real. retrieved on: May 812 24. 2018. from: www.webopedia.com.

813 C. Jose L. Flores. A. E. Gladys Cutipa. R. Lauro Enciso. 2017. Application of convolutional 814 neural networks for static hand gestures recognition under different invariant features. 815 International Conference on Electronics. Electrical Engineering and Computing: 1-4.

816 C. V Soumya. Muzameel Ahmed. 2017. Artificial Neural Network Based Identification and 817 Classification of Images of Bharatanatya Gestures. International Conference on Innovative 818 Mechanisms for Industry Applications ICIMIA: 162-166.

819 Chenyang Li. Xin Zhang. Lianwen Jin. 2017. LPSNet: A Novel Log Path Signature Feature 820 Based Hand Gesture Recognition Framework. International Conference on Computer Vision 821 Workshops ICCVW: 631-639.

822 Chenyang Zhang. Yingli Tian. Matt Huenerfauth. 2016. Multi-modality American Sign 823 Language recognition. International Conference on Image Processing ICIP: 2881-2885. 
824 Chun-Jen Tsai. Yun-Wei Tsai. Song-Ling Hsu. Ya-Chiu Wu. 2017. Synthetic Training of Deep $825 \mathrm{CNN}$ for 3D Hand Gesture Identification. International Conference on Control. Artificial 826 Intelligence. Robotics \& Optimization ICCAIRO: 165-170.

827 Danling Lu. Yuanlong Yu. Huaping Liu. 2016. Gesture Recognition Using Data Glove: An 828 Extreme Learning Machine Method. International Conference on Robotics and Biomimetics 829 ROBIO: 1349-1354.

830 David Valencia Redrovan. Donghan Kim. 2018. Hand Gestures Recognition using Machine 831 Learning for control of Multiple quadrotors. Sensors Applications Symposium SAS: 1-6.

832 Deepali N. Kakade. Prof. Dr. J.S. Chitode. 2012. Dynamic Hand Gesture Recognition : A

833 Literature Review. International Journal of Engineering Research \& Technology (IJERT): 1: 1-7.

834 Deepali Naglot. Milind Kulkarni. 2016. Real Time Sign Language Recognition using the Leap 835 Motion Controller. International Conference on Inventive Computation Technologies ICICT. 3: $836 \quad 1-5$.

837 Devendrakumar H. Pal. S. M. Kakade. 2016. Dynamic Hand Gesture Recognition Using Kinect 838 Sensor. International Conference on Global Trends in Signal Processing. Information Computing 839 and Communication ICGTSPICC: $448-453$.

840 Donq-Liang Lee. Wei-Shiuan You. 2018. Recognition of complex static hand gestures by using 841 the wristband-based contour features. IET Image Processing. 12: 80-87.

842 Eko Pramunanto. Surya Sumpeno. Rafiidha Selyna Legowo. 2017. Classification of hand gesture 843 in Indonesian sign language system using Naive Bayes. International Seminar on Sensors.

844 Instrumentation. Measurement and Metrology: 187-191.

845 Elecbits. Gesture Control Robotic Vehicle. Retrieved on: May 14, 2019. From: www. 846 elecbits.in/product/gesture-control-robotic-vehicle.

847 Erhan Akan. Hakan Tora. Baran Uslu. 2017. Hand gesture classification using inertial based 848 sensors via a neural network. International Conference on Electronics. Circuits and Systems: 849 140-143.

850 Fabian Sachara. Thomas Kopinski. Alexander Gepperth. Uwe Handmann. 2017. Free-hand 851 Gesture Recognition with 3D-CNNs for In-car Infotainment Control in Real-time. International 852 Conference on Intelligent Transportation Systems ITSC: 959-964.

853 Fuchang Yang. Hijian Shi. 2016. Research on Static Hand Gesture Recognition Technology for 854 Human Computer Interaction System. International Conference on Intelligent Transportation. 855 Big Data \& Smart City: 459-463.

856 Gi Hyun Lim. Eurico Pedrosa. Filipe Amaral. Nuno Lau. Artur Pereira. José Luís Azevedo. 857 Bernardo Cunha. 2017. Neural regularization jointly involving neurons and connections for 858 robust image classification. International Conference on Multisensor Fusion and Integration for 859 Intelligent Systems MFI: 336-341. 
860 Hafiz Mohsin Abdul-Rashid. Lehmia Kiran. M. Danish Mirrani. M. Noman Maraaj. 2017. 861 CMSWVHG-control MS Windows via hand gesture. International Multi-topic Conference 862 INMIC: $1-7$.

863 Haitham Badi. Alaa Hamza. Sabah Hasan. 2017. New Method for Optimization of Static Hand 864 Gesture Recognition. Intelligent Systems Conference IntelliSys: 542-544.

865 Hang Zhao. Jiangping Hu. Yuping Zhang. Hong Cheng. 2017. Hand Gesture Based Control 866 Strategy for Mobile Robots. Chinese Control and Decision Conference CCDC: 5868-5872.

867 Hari Prabhat Gupta. Haresh S. Chudgar. Siddhartha Mukherjee. Tanima Dutta. Kulwant Sharma. 868 2016. A Continuous Hand Gestures Recognition Technique for Human-Machine Interaction 869 Using Accelerometer and Gyroscope Sensors. Sensors Journal. 16: 6425-6432.

870 Hexa Research. 2017. Gesture Recognition Market Analysis by Technology 2D. 3D. By 871 Application Tablets \& Notebooks. Smartphones. Gaming Consoles. Smart Televisions. Laptops 872 \& Desktops and Segment Forecasts. 2014 - 2024: 1-100.

873 Himadri Nath Saha. Shinjini Ray. Sudipta Saha. Sayan Tapadar. and Suhrid Krishna Chatterjee. 874 2018. A Machine Learning Based Approach for Hand Gesture Recognition using Distinctive 875 Feature Extraction. Computing and Communication Workshop and Conference: 91-98.

876 Ibrahim Alnujaim. Hashim Alali. Faisal Khan. Youngwook Kim. 2018. Hand Gesture

877 Recognition Using Input Impedance Variation of Two Antennas with Transfer Learning. Sensors 878 Journal. 18: 4129-4135.

879 Isack Bulugu. Zhongfu Ye. Jamal Banzi. 2017. Higher-Order Local Autocorrelation Feature 880 Extraction Methodology for Hand Gestures Recognition. International Conference on 881 Multimedia and Image Processing ICMIP: 83-87.

882 Jakub Tomczyński. Tomasz Mańkowski. Piotr Kaczmarek. 2017. Influence of sEMG electrode 883 matrix configuration on hand gesture recognition performance. Signal Processing: Algorithms. 884 Architectures. Arrangements. and Applications SPA: 42-47.

885 Javier Orlando Pinzón Arenas. Paula Catalina Useche Murillo. Robinson Jiménez Moreno. 2017. 886 Convolutional neural network architecture for hand gesture recognition. International Conference 887 on Electronics. Electrical Engineering and Computing: 1-6.

888 Jessie R. Balbin. Dionis A. Padilla. Felicito S. Caluyo. Janette C. Fausto. Carlos C. Hortinela. 889 Cyrel O. Manlises. Christine Kate S. Bernardino. Ezra G. Fiñones. Lanuelle T. Ventura. 2016. 890 Sign Language Word Translator Using Neural Networks for the Aurally Impaired as a Tool for 891 Communication. International Conference on Control System. Computing and Engineering 892 ICCSCE: 425-429.

893 Jiajun Zhang. Zhiguo Shi. 2017. Deformable deep convolutional generative adversarial network 894 in microwave based hand gesture recognition system. International Conference on Wireless 895 Communications and Signal Processing WCSP: 1-6. 
896 Jian Zhao. Jingna Mao. Guijin Wang. Huazhong Yang. and Bo Zhao. 2017. A Miniaturized 897 Wearable Wireless Hand Gesture Recognition System Employing Deep-Forest Classifier. 898 Biomedical Circuits and Systems Conference: 1-4.

899 Jinxing Yang. Jianhong Pan. Jun Li. 2017. sEMG-Based Continuous Hand Gesture Recognition 900 Using GMM-HMM and Threshold Model. International Conference on Robotics and 901 Biomimetics ROBIO: 1509-1514.

902 Jozef Goga. Slavomír Kajan. 2017. Hand gesture recognition using 3D sensors. International 903 Symposium ELMAR: 181-184.

904 Karthik Sivarama Krishnan. Akash Saha. Srinath Ramachandran. Shitij Kumar. 2017. 905 Recognition of Human Arm Gestures Using Myo Armband for the Game of Hand Cricket. 906 International Symposium on Robotics and Intelligent Sensors IRIS: 389-394.

907 Kinect gestures. Retrieved on: May 14, 2019. From: www.support.xbox.com/en-TT/xbox908 360/kinect/body-controller.

909 Kuang-Yow Lian. Chun-Chieh Chiu. Yong-Jie Hon. Wen-Tsai Sung. 2017. Wearable armband 910 for real time hand gesture recognition. International Conference on Systems. Man. and 911 Cybernetics: 2992-2995.

912 L. P Madushanka. R. G. D. C Senevirathne. L. M. H Wijesekara. S. M. K. D Arunatilake. K. D 913 Sandaruwan. 2016. Framework for Sinhala Sign Language Recognition and Translation Using a 914 Wearable Armband. International Conference on Advances in ICT for Emerging Regions ICTer: 915 49-57.

916 Lalit Kane. Pritee Khanna. 2017. Vision-Based Mid-Air Unistroke Character Input Using Polar 917 Signatures. Transactions on Human-Machine Systems. 47: 1077-1088.

918 Laura Dipietro. Angelo M. Sabatini. and Paolo Dario. 2008. A Survey of Glove-Based Systems 919 and Their Applications. Transactions on Systems. Man. And Cybernetic. 38: 461-480.

920 Marco E. Benalcázar. Andrés G. Jaramillo. Jonathan. A. Zea. Andrés Páez. Víctor Hugo 921 Andaluz. 2017. Hand gesture recognition using machine learning and the Myo armband. 922 European Signal Processing Conference: 1040-1044.

923 Marco E. Benalcázar. Cristhian Motoche. Jonathan A. Zea. Andrés G. Jaramillo. Carlos E. 924 Anchundia. Patricio Zambrano. Marco Segura. Freddy Benalcázar Palacios. María Pérez. 2017.

925 Real-time hand gesture recognition using the Myo armband and muscle activity detection.

926 Second Ecuador Technical Chapters Meeting ETCM: 1-6.

927 Margaret Rouse. 2016. Analytics tools help make sense of big data. AWS. retrieved on: May 24. 928 2018. from: searchbusinessanalytics.techtarget.com.

929 Marlon Oliveira. Alistair Sutherland. Mohamed Farouk. 2016. Two-stage PCA with Interpolated 930 Data for Hand Shape Recognition in Sign Language. Applied Imagery Pattern Recognition 931 Workshop AIPR: 1-4. 
932 Meenakshi Panwar. Pawan Singh Mehra. 2011. Hand gesture recognition for human computer 933 interaction. International Conference on Image Information Processing: 1-7.

934 Moher D, Liberati A, Tetzlaff J, Altman DG. 2009. PRISMA Group. Preferred reporting items 935 for systematic reviews and meta-analyses: the PRISMA statement. PLOS Medicine 6: e1000097.

936 Mohini Gupta. R. Rohini. P.E.V.V Reddy. P. Bhanu Prakash. K.T.P.S Kumar. 2015.

937 International Journal of Engineering Trends and Technology IJETT. 21: 229-232.

938 Nabeel Siddiqui. Rosa H. M. Chan. 2017. A Wearable Hand Gesture Recognition Device Based 939 on Acoustic Measurements at Wrist. International Conference of the IEEE Engineering in 940 Medicine and Biology Society EMBC: 4443-4446.

941 Nilima Mansing Patil. S. R. Patil. 2017. Review on real-time EMG acquisition and hand gesture 942 recognition system. International conference of Electronics. Communication and Aerospace 943 Technology ICECA: 694-696.

944 Oinam Robita Chanu. Anushree Pillai. Spandan Sinha. Piyanka Das. 2017. Comparative Study 945 for Vision Based and Data Based Hand Gesture Recognition Technique. International 946 Conference on Intelligent Communication and Computational Techniques: 26-31.

947 Oyndrila De. Puskar Deb. Sagnik Mukherjee. Sayantan Nandy. Tamal Chakraborty. Sourav 948 Saha. 2016. Computer Vision Based Framework for Digit Recognition by Hand Gesture 949 Analysis. Information Technology. Electronics and Mobile Communication Conference 950 IEMCON: 1-5.

951 P. D. S. H. Gunawardane. Nimali T. Medagedara. 2017. Comparison of hand gesture inputs of 952 leap motion controller \& data glove in to a soft finger. International Symposium on Robotics and 953 Intelligent Sensors IRIS: 62-68.

954 Peijun Bao. Ana I. Maqueda. Carlos R. del-Blanco. Narciso García. 2017. Tiny Hand Gesture 955 Recognition without Localization via a Deep Convolutional Network Transactions on Consumer 956 Electronics. 63: 251-257.

957 Piotr Kaczmarek. Tomasz Mańkowski. Jakub Tomczyński. 2017. Towards sensor position958 invariant hand gesture recognition using a mechanomyographic interface. Signal Processing: 959 Algorithms. Architectures. Arrangements. and Applications SPA: 53-58.

960 Prashan Premaratne. 2014. Historical Development of Hand Gesture Recognition. Cognitive 961 Science and Technology book series CSAT. Springer: 5-29.

962 Praveen Kumar Sharma. Shreya Sharma. 2015. Evolution of Hand Gesture Recognition: A 963 Review. International Journal of Engineering And Computer Science. 4: 9962-9965.

964 Qingrui Zhang. Mingqiang Yang. Qinghe Zheng. Xinxin Zhang. 2017. Segmentation of Hand 965 Gesture Based on Dark Channel Prior in Projector-Camera System. International Conference on 966 Communications in China ICCC: 1-6. 
967 Rafiqul Zaman Khan. Noor Adnan Ibraheem. 2012. Hand Gesture Recognition: A Literature

968 Review. International Journal of Artificial Intelligence \& Applications (IJAIA): 3:161-174.

969 Rajeshree Rokade-Shinde. Jayashree Sonawane. 2016. Dynamic Hand Gesture Recognition.

970 International Conference on Signal and Information Processing IConSIP: 1-6.

971 Rania A. Elsayed. Mohammed S. Sayed. Mahmoud I. Abdalla. 2017. Hand Gesture Recognition

972 Based on Dimensionality Reduction of Histogram of Oriented Gradients. Japan-Africa

973 Conference on Electronics. Communications and Computers JAC-ECC: 119-122.

974 Rasel Ahmed Bhuiyan. Abdul Kawsar Tushar. Akm Ashiquzzaman. Jungpil Shin. Md Rashedul

975 Islam. 2017. Reduction of gesture feature dimension for improving the hand gesture recognition

976 performance of numerical sign language. International Conference of Computer and Information

977 Technology ICCIT: 1-6.

978 Rishabh Sharma. Raj Shikher. Nutan V. Bansode. Prachi R. Rajarapollu. 2016. Interactive

979 Projector Screen with Hand Detection Using Gestures. International Conference on Automatic

980 Control and Dynamic Optimization Techniques ICACDOT: 574-577.

981 Riya Bora. Ankita Bisht. Aradhya Saini. Tanu Gupta. Ankush Mittal. 2017. ISL Gesture

982 Recognition Using Multiple Feature Fusion. International Conference on Wireless

983 Communications. Signal Processing and Networking WiSPNET: 196-199.

984 Rokhsana Titlee. Ashfaq Ur Rahman. Hasan U. Zaman. Hafiz Abdur Rahman. 2017. A novel

985 design of an intangible hand gesture controlled computer mouse using vision based image

986 processing. International Conference on Electrical Information and Communication Technology

987 EICT: 1-4.

988 Rosalina. Lita Yusnita. Nur Hadisukmana. R.B Wahyu. Rusdianto Roestam. Yuyu Wahyu. 2017. 989 Implementation of Real-Time Static Hand Gesture Recognition using Artificial Neural Network. 990 Computer Applications and Information Processing Technology: 1-6.

991 Rytis Augustauskas. Arunas Lipnickas. 2017. Robust hand detection using arm segmentation

992 from depth data and static palm gesture recognition. International Conference on Intelligent Data

993 Acquisition and Advanced Computing Systems: Technology and Applications: 664-667.

994 S. Reshna. M. Jayaraju. 2017. Spotting and Recognition of Hand Gesture for Indian Sign

995 Language Recognition System with Skin Segmentation and SVM. International Conference on

996 Wireless Communications. Signal Processing and Networking WiSPNET: 386-390.

997 S. Siji Rani. K. J. Dhrisya. M. Ahalyadas. 2017. International Conference on Advances in

998 Computing. Communications and Informatics ICACCI: 1500-1505.

999 Said Yacine Boulahia. Eric Anquetil. Franck Multon. Richard Kulpa. 2017. Dynamic hand 1000 gesture recognition based on 3D pattern assembled trajectories. International Conference on 1001 Image Processing Theory. Tools and Applications IPTA: 1-6.

1002 Samata Mutha. Dr. K.S.Kinage. 2015. Study on Hand Gesture Recognition. International Journal 1003 of Computer Science and Mobile Computing. 4: 51-57. 
1004 Shaun Canavan. Walter Keyes. Ryan Mccormick. Julie Kunnumpurath. Tanner Hoelzel. Lijun 1005 Yin. 2017. Hand gesture recognition using a skeleton-based feature representation with a random 1006 regression forest. International Conference on Image Processing ICIP: 2364-2368.

1007 Shengchang Lan. Zonglong He. Haoyu Tang. Kai Yao. Wenshuang Yuan. 2017. A Hand Gesture 1008 Recognition System Based on 24GHz Radars. International Symposium on Antennas and 1009 Propagation ISAP: 1-2.

1010 Shome Subhra Das. 2017. Detection of Self Intersection in Synthetic Hand Pose Generators. 1011 International Conference on Machine Vision Applications MVA: 354-357.

1012 Shunzhan He. Chenguang Yang. Min Wang. Long Cheng. Zedong Hu. 2017. Hand Gesture 1013 Recognition using MYO Armband. Chinese Automation Congress CAC: 4850-4855.

1014 Sih-Huei Chen. Ari Hernawan. Yuan-Shan Lee. Jia-Ching Wang. 2017. Hand gesture 1015 recognition based on Bayesian sensing hidden Markov models and Bhattacharyya divergence. 1016 International Conference on Image Processing ICIP: 3535-3539.

1017 Soukaina Chraa Mesbahi. Mohamed Adnane Mahraz. Jamal Riffi. Hamid Tairi. 2018. Hand 1018 gesture recognition based on convexity approach and background subtraction. International 1019 Conference on Intelligent Systems and Computer Vision ISCV: 1-5.

1020 Srinidhi Hegde. Ramakrishna Perla. Ramya Hebbalaguppe. Ehtesham Hassan. 2016. GestAR: 1021 Real Time Gesture Interaction for AR with Egocentric View. International Symposium on Mixed 1022 and Augmented Reality ISMAR-Adjunct: 262-267.

1023 Stefano Sapienza. Paolo Motto Ros. David Alejandro Fernandez Guzman. Fabio Rossi. Rossana 1024 Terracciano. Elisa Cordedda. Danilo Demarchi. 2018. On-Line Event-Driven Hand Gesture 1025 Recognition Based on Surface Electromyographic Signals. International Symposium on Circuits 1026 and Systems ISCAS: 1-5.

1027 Sungho Shin. Wonyong Sung. 2016. Dynamic Hand Gesture Recognition for Wearable Devices 1028 with Low Complexity Recurrent Neural Networks. International Symposium on Circuits and 1029 Systems ISCAS: 2274-2277.

1030 T. Vedha Viyas. R. Willbert Baskar. N. Simrose Gabriel. A. Sanjive. 2017. Hand Pantomime 1031 Apperception For Robotic Arm Control. International conference of Electronics. Communication 1032 and Aerospace Technology ICECA. 2: 120-125.

1033 Takuya Sakamoto. Xiaomeng Gao. Ehsan Yavari. Ashikur Rahman. Olga Boric-Lubecke. Victor 1034 M. Lubecke. 2017. Radar-based hand gesture recognition using I-Q echo plot and convolutional 1035 neural network. Conference on Antenna Measurements \& Applications CAMA: 393-395.

1036 Tejashree P. Salunke S .D. Bharkad. 2017. Power Point Control Using Hand Gesture 1037 Recognition Based on Hog Feature Extraction And K-NN Classification. International 1038 Conference on Computing Methodologies and Communication: 1151-1155. 
1039 Vaibhavi S. Gandhi. Akshay A. Khond. Sanket N. Raut. Vaishali A. Thakur. Shabnam S. Shaikh.

1040 2014. A Review of Various Gesture Recognition Techniques. International Journal of

1041 Engineering And Computer Science. 3: 8202-8206.

1042 Vimonhak Sombandith. Aranya Walairacht. Somsak Walairacht. 2017. Hand gesture recognition

1043 for Lao alphabet sign language using HOG and correlation. International Conference on

1044 Electrical Engineering/Electronics. Computer. Telecommunications and Information

1045 Technology: 649-651.

1046 Vivek D. Lad. Ramesh M. Kagalkar. 2017. Methodology for Real Time Hand Gesture

1047 Recognition and Generating Text Description Using Histogram Techniques. International

1048 Conference on Intelligent Computing and Control I2C2: 1-7.

1049 Vladislava Bobić. Predrag Tadić. Goran Kvaščev. 2016. Hand gesture recognition using neural

1050 network based techniques. Symposium on Neural Networks and Applications NEUREL: 1-4.

1051 Washef Ahmed. Kunal Chanda. Soma Mitra. 2016. Vision Based Hand Gesture Recognition

1052 Using Dynamic Time Warping for Indian Sign Language. International Conference on

1053 Information Science ICIS: 120-125.

1054 Weiguo Zhou. Congyi Lyu. Xin Jiang. Peng Li. Haoyao Chen. Yun-Hui Liu. 2017. Real-time

1055 Implementation of Vision-based Unmarked Static Hand Gesture Recognition with Neural

1056 Networks based on FPGAs. International Conference on Robotics and Biomimetics ROBIO:

1057 1026-1031.

1058 Xi Liu. Chen Li. Lihua Tian. 2017. Hand Gesture Recognition based on Wavelet Invariant

1059 Moments International Symposium on Multimedia ISM: 459-464.

1060 Xiang Gao. Lei Shi. Qiang Wang. 2017. The design of robotic wheelchair control system based 1061 on hand gesture control for the disabled. International Conference on Robotics and Automation 1062 Sciences: 30-34.

1063 Xinghao Chen. Hengkai Guo. Guijin Wang. Li Zhang. 2017. Motion feature augmented 1064 recurrent neural network for skeleton-based dynamic hand gesture recognition. International 1065 Conference on Image Processing ICIP: 2881-2885.

1066 Yifan Zhang. Congqi Cao. Jian Cheng. Hanqing Lu. 2018. EgoGesture: A New Dataset and 1067 Benchmark for Egocentric Hand Gesture Recognition. Transactions on Multimedia. 20: 103810681050.

1069 Yiwen He. Jianyu Yang. Zhanpeng Shao. Youfu Li. 2017. Salient Feature Point Selection for 1070 Real Time RGB-D Hand Gesture Recognition. International Conference on Real-time 1071 Computing and Robotics RCAR: 103-108.

1072 Yu Qiao. Zhiquan Feng. Xiaoyan Zhou. Xiaohui Yang. 2017. Principle Component Analysis 1073 Based Hand Gesture Recognition for Android Phone Using Area Features. International 1074 Conference on Multimedia and Image Processing ICMIP: 108-112. 
1075 Yuefeng Li. Keshi He. Xueli Sun. Honghai Liu. 2016. Human-machine Interface Based on 1076 Multi-channel Single-element Ultrasound Transducers: A Preliminary Study. International 1077 Conference on e-Health Networking. Applications and Services Healthcom: 1-6.

1078 Yuhui Zhu. Shuo Jiang. Peter B. Shull. 2018. Wrist-worn hand gesture recognition based on 1079 barometric pressure sensing. International Conference on Wearable and Implantable Body 1080 Sensor Networks BSN: 181-184.

1081 Yuntao Ma. Yuxuan Liu. Ruiyang Jin. Xingyang Yuan. Raza Sekha. Samuel Wilson. Ravi 1082 Vaidyanathan. 2017. Hand gesture recognition with convolutional neural networks for the 1083 multimodal UAV control: 198-203.

1084 Yuta Sugiura. Fumihiko Nakamura. Wataru Kawai. Takashi Kikuchi. Maki Sugimoto. 2017. 1085 Behind the palm: Hand gesture recognition through measuring skin deformation on back of hand 1086 by using optical sensors. Conference of the Society of Instrument and Control Engineers of 1087 Japan: 1082-1087.

1088 Zengshan Tian. Jiacheng Wang. Xiaolong Yang. Mu Zhou. 2018. WiCatch: A Wi-Fi Based 1089 Hand Gesture Recognition System Access. 6: 16911-16923.

1090 Zhiwen Lei. Xiaoxiao Yang. Yanzhou Gong. Weixing Huang. Jian Wang. Guigang Zhang. 2017. 1091 A Robust Hand Cursor Interaction Method Using Kinect. International Symposium on 1092 Multimedia ISM: 543-548. 


\section{Table $\mathbf{1}$ (on next page)}

Classification of Extracted Papers 


\begin{tabular}{|l|l|l|}
\hline Topic & Sub-Topics & References Count \\
\hline \multirow{4}{*}{$\begin{array}{l}\text { Hechniques } \\
\text { Techture }\end{array}$} & $\begin{array}{l}\text { Gesture Acquisition } \\
\text { Methods }\end{array}$ & 58 \\
\cline { 2 - 3 } & Feature Extraction & 51 \\
\cline { 2 - 3 } & $\begin{array}{l}\text { Classification of Hand } \\
\text { Gestures }\end{array}$ & 71 \\
\hline $\begin{array}{l}\text { Hand Gesture } \\
\text { Recognition } \\
\text { Applications }\end{array}$ & Sign Language & 22 \\
\cline { 2 - 3 } $\begin{array}{l}\text { Hand Gesture } \\
\text { Recognition } \\
\text { Challenges }\end{array}$ & Robotics & 11 \\
\cline { 2 - 3 } & Others & 20 \\
\cline { 2 - 3 } & Surroundings & 18 \\
\cline { 2 - 3 } & Training and Testing Data & 13 \\
\hline
\end{tabular}

1 
Figure 1

Figure 1 PRISMA flow diagram of the study selection process

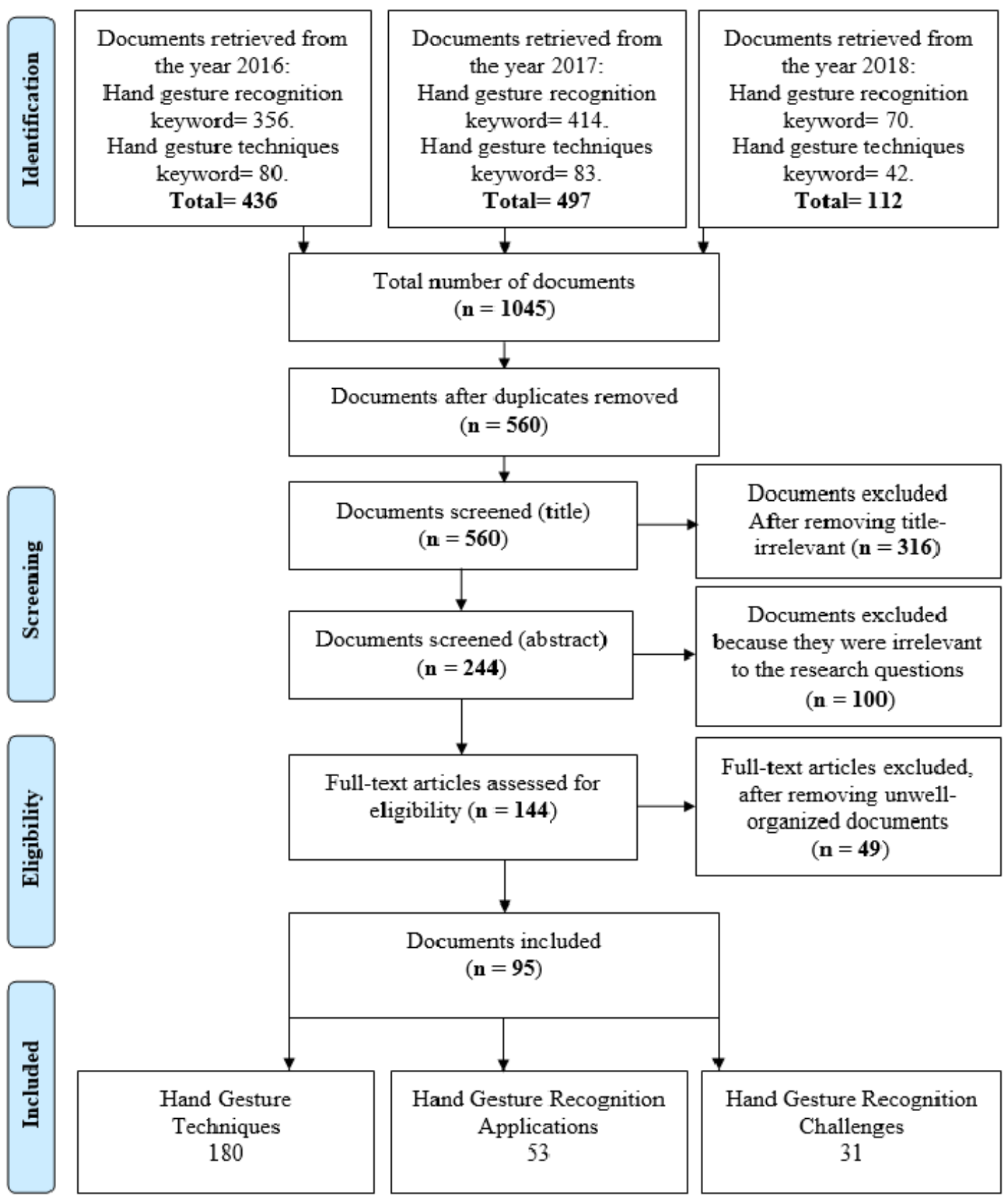


Figure 2

Figure 2 Examples of Hand Gestures in Sign Language

(Rosalina et al., 2017)
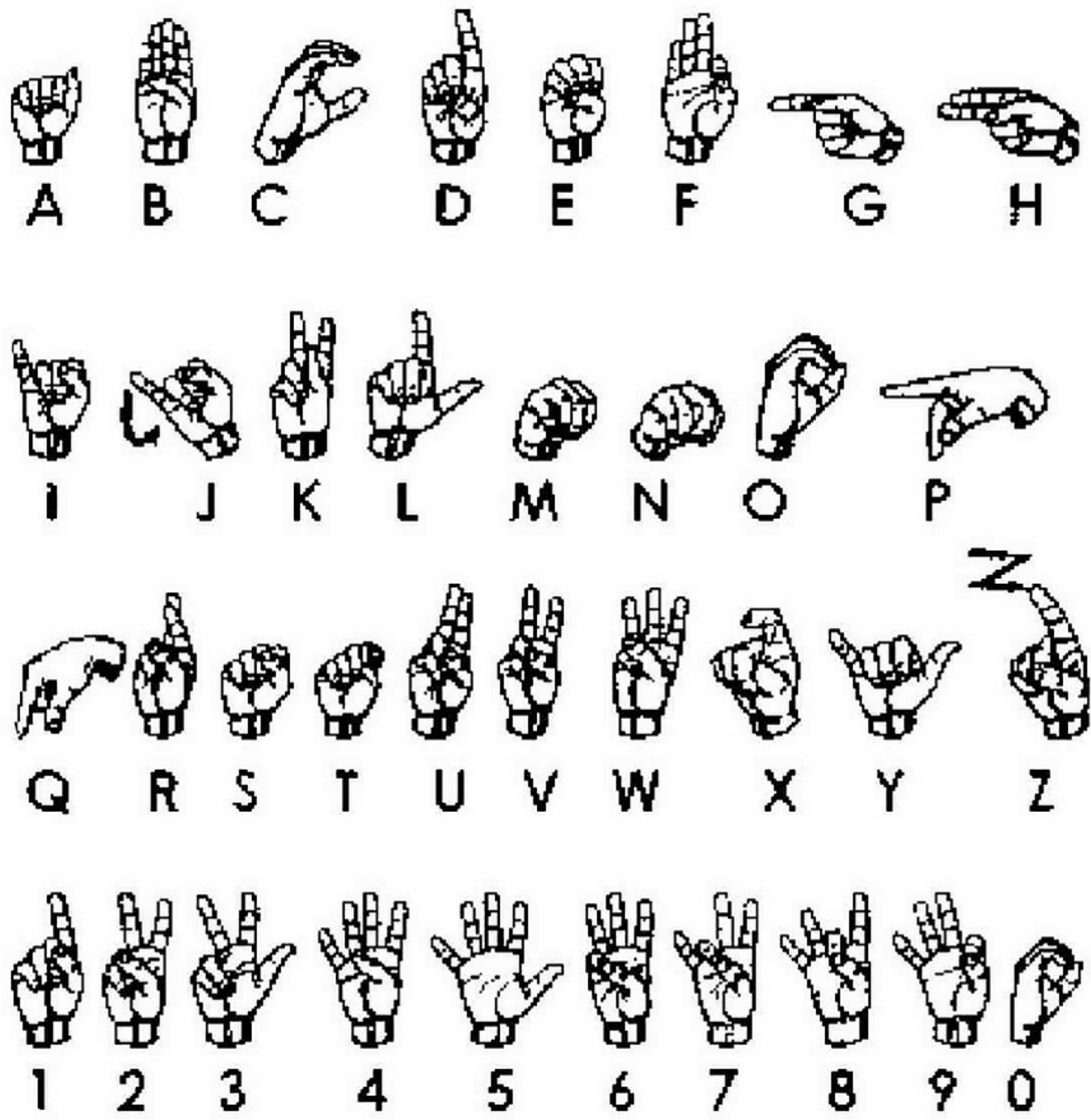
Figure 3

Figure 3 The Basic Steps of Hand Gesture Recognition

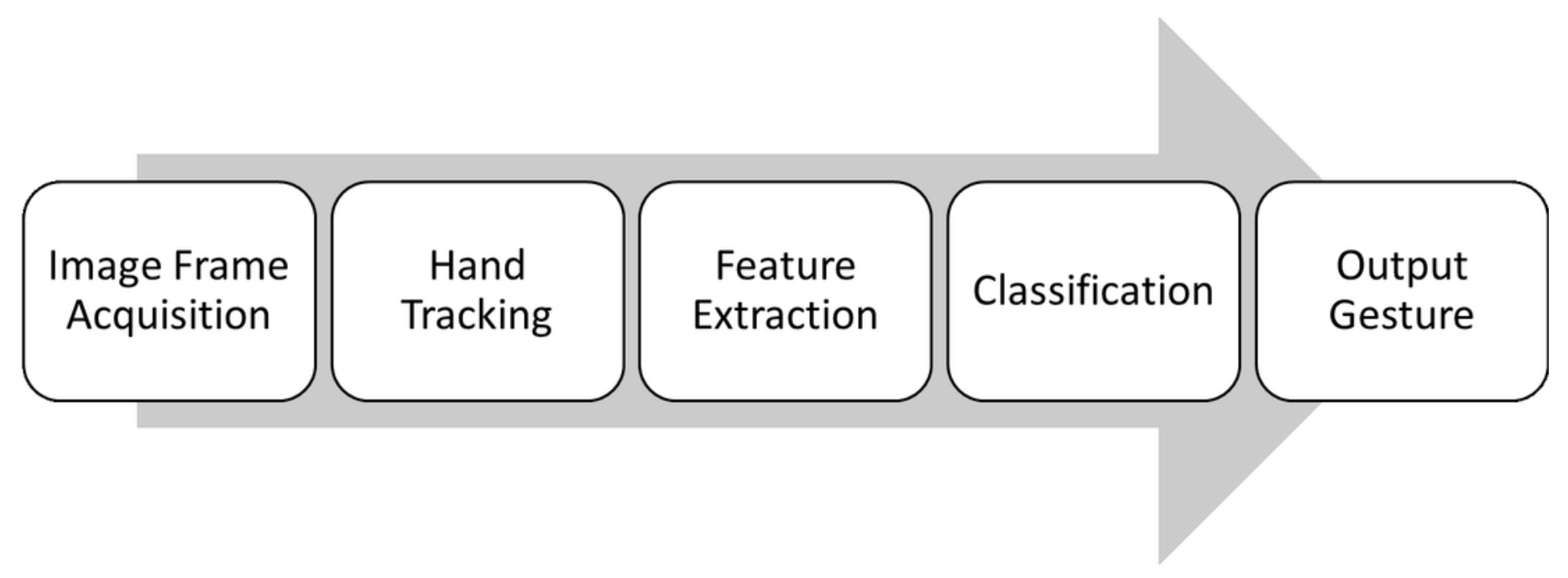


Figure 4

Figure 4 Results

Each research question is illustrated in this figure, along with the subcategories suggested and the resulting most common technique or problem in them.

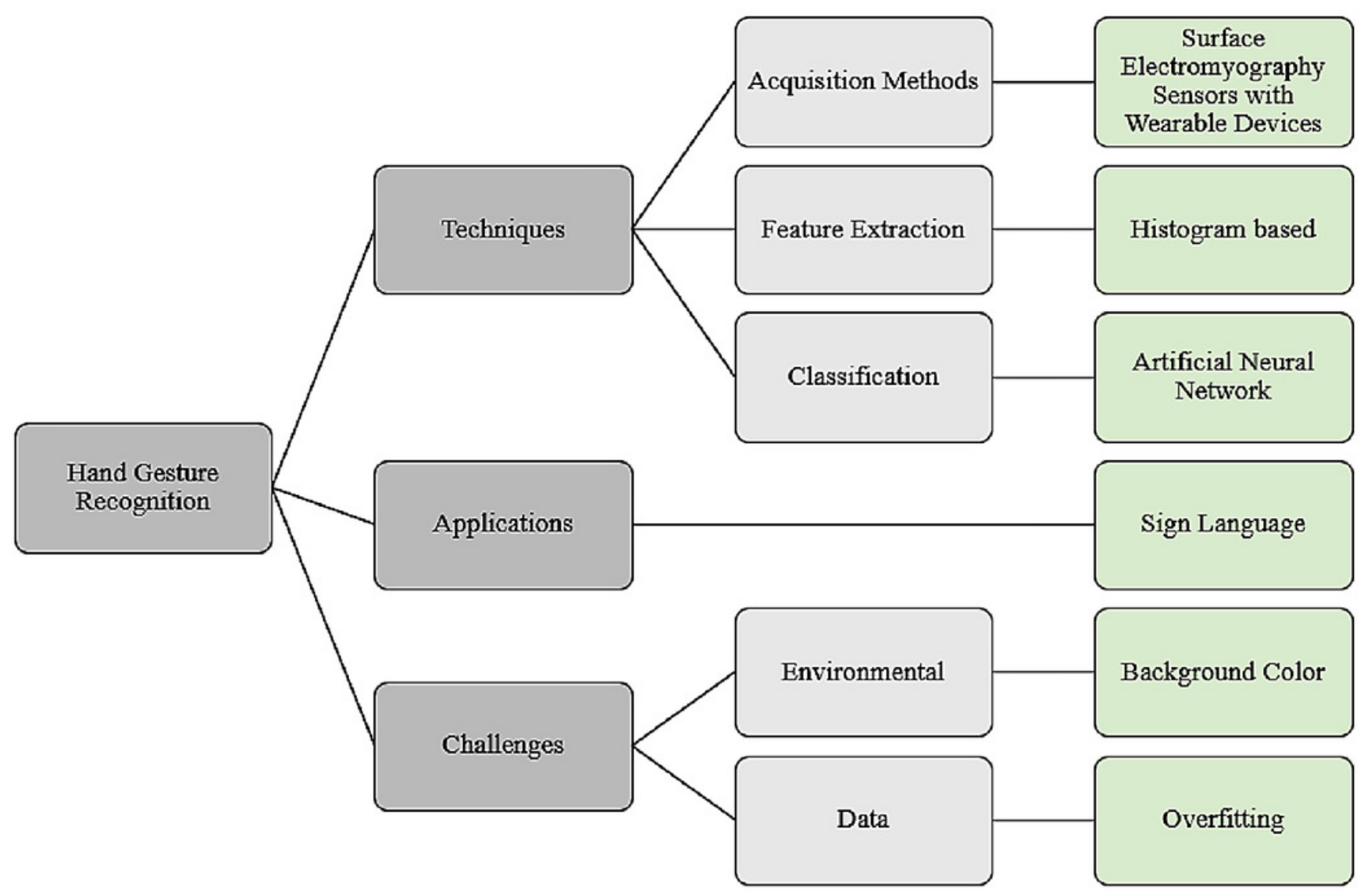




\section{Figure 5}

Figure 5 Example of Hand Gesture Recognition using Kinect

(Kinect, 2019)

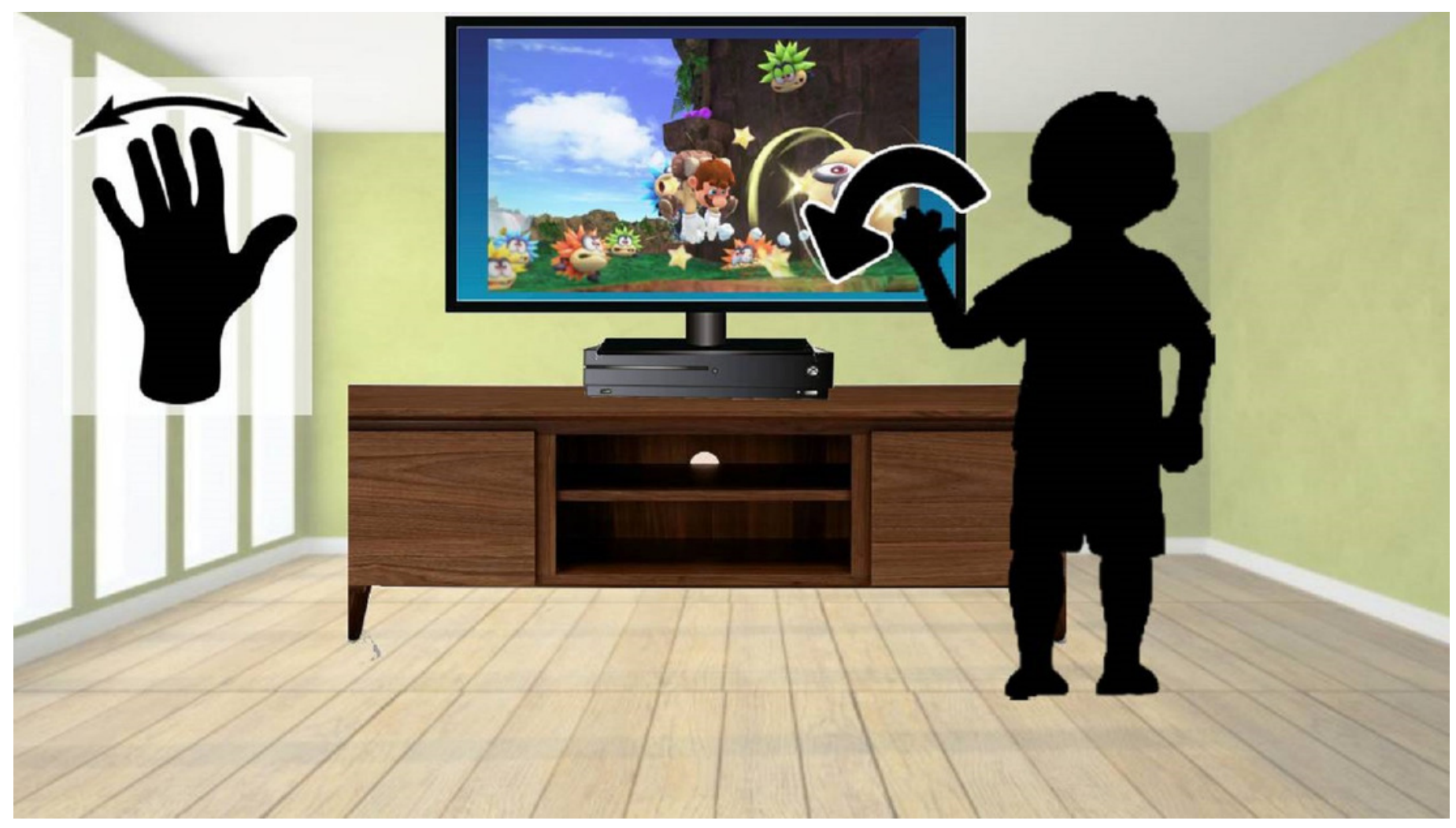




\section{Figure 6}

Figure 6 Example of the Use of Hand Gestures in Robotics

(elecbits, 2019)

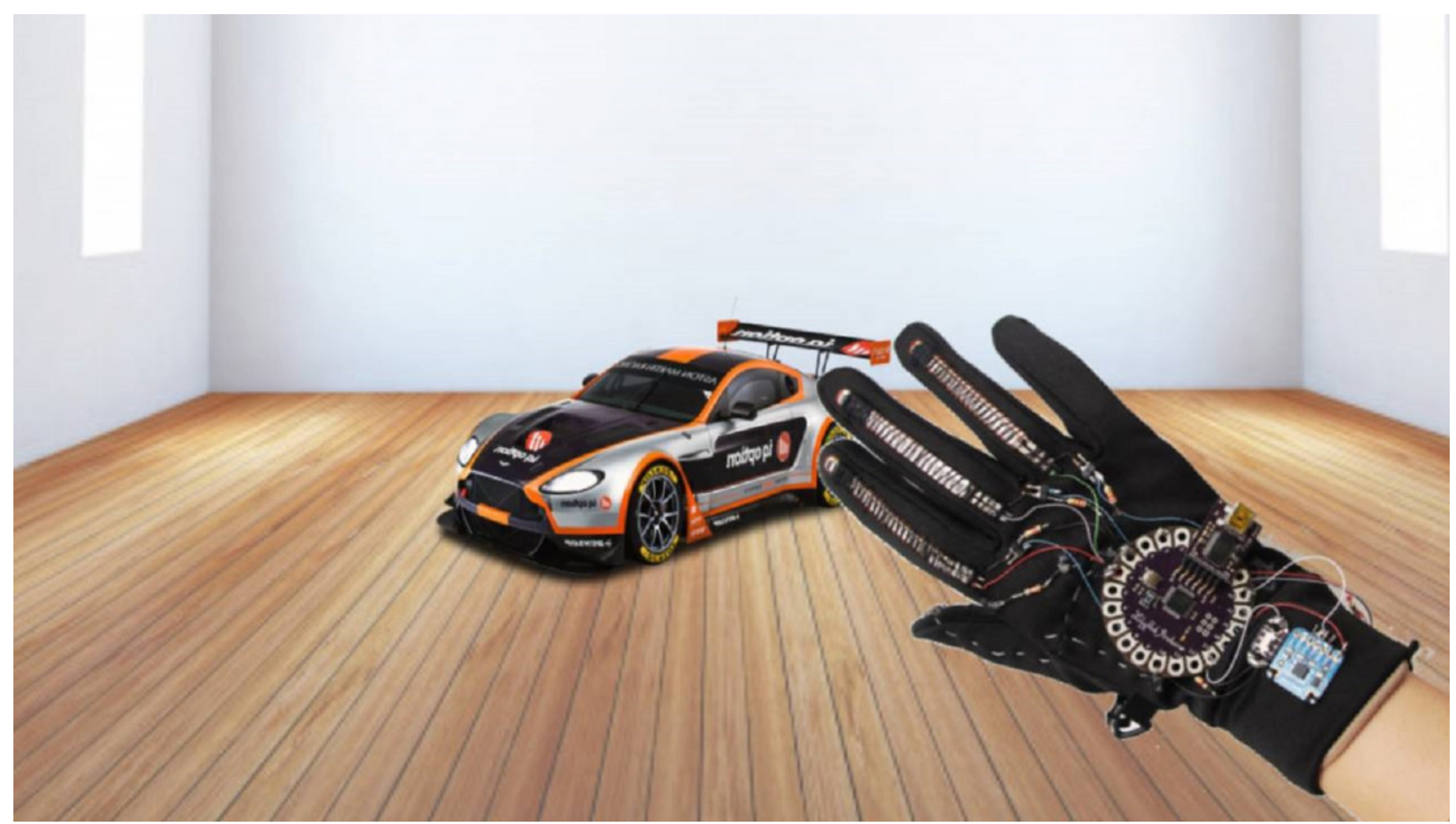

